\title{
A Turbulent History
}

\begin{abstract}
Large solar energetic-particle (SEP) events are clearly associated in time with eruptive phenomena on the Sun, but how? When large SEP events were first observed, flares were the only visible candidate, and diffusion theory was stretched to explain how the particles could spread through space, as widely as observed. The observation of coronal mass ejections (CMEs), and the wide, fast shock waves they can drive, provided better candidates later. Then small events were found with 1000 -fold enhancements in ${ }^{3} \mathrm{He} /{ }^{4} \mathrm{He}$ that required a different kind of source-should we reconsider flares, or their open-field cousins, solar jets? The ${ }^{3} \mathrm{He}$-rich events were soon associated with the electron beams that produce type III radio bursts. It seems the radio astronomers knew of both SEP sources all along. Sometimes the distinction between the sources is blurred when shocks reaccelerate residual ${ }^{3} \mathrm{He}$-rich impulsive suprathermal ions. Eventually, however, we would even begin to measure the source-plasma temperature that helps to better distinguish the SEP sources.
\end{abstract}

The first reported observation of a solar flare, that of 1118 GMT on 1 September, 1859, was published by a self-established astronomer Richard Carrington (1860) who saw the brightening of a white-light solar flare, which lasted over $5 \mathrm{~min}$, while he was observing sunspots. The observation was confirmed by his friend Richard Hodgson. Carrington noted that the brightening did not seem to disrupt the underlying structure at all. However, apparently-associated geomagnetic effects were also noticed. 


\subsection{The First SEPs}

Some 87 years later Scott Forbush (1946) reported the first SEPs as an increase in what we now call a ground-level event (GLE). Protons of GeV energies cause nuclear cascades through the atmosphere. Forbush was observing the intensities of similar secondary particles produced by galactic cosmic rays (GCRs) using groundlevel ion chambers and especially the "Forbush decreases" now known to be caused by ejecta from the Sun whose shielding reduces the intensities of the GCRs. Three large solar events beginning in February and March 1942 produced sharp intensity increases from SEPs prior to the Forbush decreases. Since Forbush was unaware of CMEs and the shock waves they drive, it was natural for him to assume that the SEPs had come from the associated flares, which could even be seen.

The nuclear cascade from the large GLE of 23 February 1956 was measured by 6 neutron detectors widely spaced in geolatitude, and a balloon-borne detector which measured the atmospheric absorption mean free path of the solar protons (Meyer et al. 1956). The SEP increase immediately preceded a Forbush decrease in GCRs that these authors regarded as a chance coincidence.

Since 1956, ground-level neutron monitors have held the promise of using the different geomagnetic cutoff rigidities at multiple sites to measure the high-energy proton spectra. Over 70 GLEs have been recorded in over 70 years (Cliver et al. 1982; Cliver 2006; Gopalswamy et al. 2012) but most of them barely rise above the GCRs. It is only recently that the neutron-monitor measurements, combined with satellite measurements have finally begun to yield rigidity spectra for 53 of the GLEs (Tylka and Dietrich 2009) as we will see in Sect. 6.1.

\subsection{Solar Radio Bursts and Electrons}

Much more sensitive ground-based evidence of SEPs was derived from the radio emission caused by streaming energetic electrons. As electrons of 10-100 keV stream out along magnetic fields from sources near the Sun, they excite Langmuir wave oscillations at the local plasma frequency. Since the plasma frequency depends upon the square root of the local plasma electron density, the emission, called a type III burst (e.g. Thejappa et al. 2012), drifts rapidly lower in frequency across the metric radio band as the electrons stream out from the Sun. At shock waves, electrons accelerated in the $\boldsymbol{V}_{\mathbf{S}} \times \boldsymbol{B}$ electric field similarly excite local oscillations producing a type II burst (e.g. Ganse et al. 2012), but since the electrons are carried downstream of the shock soon after acceleration, the emission only drifts out with the shock speed, $V_{\mathrm{S}}$, i.e. much more slowly.

In their review of the status of solar radio measurements Wild et al. (1963) identified two sites of acceleration near the Sun:

- Impulsive bursts of electrons were accelerated to produce type III radio bursts.

- Protons were accelerated at shock waves where accompanying electrons generated type II radio bursts. 
After measurements in space became possible, Lin (1970, 1974) distinguished SEP events with $40 \mathrm{keV}$ electrons that were associated with type III radio bursts, optical flares, and 20-keV X-ray bursts. These differed from the large proton events in which the accompanying electrons were mainly relativistic. Lin identified "pure" impulsive electron events, meaning events in which any accompanying ions were not yet detectible, at that time. The direct measurements of electrons by Lin supported the ideas of Wild et al. (1963).

An example of a modern space-based measurement of the dynamic radio spectra of type II and type III radio bursts from the Wind/WAVES instrument (Bougeret et al. 1995; https://ssed.gsfc.nasa.gov/waves/index.html) from a small event is shown in the lower panel of Fig. 2.1. The plasma frequency decreases with distance from the Sun as $\sqrt{ } n_{e}$ where $n_{e}$ is the local plasma electron density. Ground-based radio instruments can measure only high frequencies produced near the Sun while space-based instruments cover sources moving from the Sun to the Earth. For type III bursts, frequencies drift rapidly, produced by $10-100 \mathrm{keV}$ electrons streaming out from the Sun; frequencies in type II bursts drift outward from a source moving at the speed $\left(\sim 1000 \mathrm{~km} \mathrm{~s}^{-1}\right)$ of a shock wave. In some events, type IV emission occupies the frequency region after and above the type II burst. Type IV emission may be produced by electrons accelerated on the sunward flanks of the shock or by reconnection regions in or behind the CME.

The upper panel in Fig. 2.1 shows a type II burst propagating from the groundbased metric $(\geq 25 \mathrm{MHz})$ regime to the decametric-hectometric ( $\mathrm{DH}, 1-14 \mathrm{MHz}$ ) regime at $\sim 3 \mathrm{R}_{\mathrm{S}}$. Cliver et al. (2004) found a $90 \%$ association of DH type-II bursts with SEP events with $20 \mathrm{MeV}$ protons but only $25 \%$ for metric type-II bursts without $\mathrm{DH}$ emission. This suggested shock acceleration that was strongest above $\sim 3 \mathbf{R}_{\mathbf{S}}$. Strong shocks that survive beyond $\sim 3 \mathrm{R}_{\mathrm{S}}$ are more likely to have expanded to a broad longitude extent.

Thus, prior to observations in space, there were observations of rare GLEs produced by $\mathrm{GeV}$ protons, and much-more-sensitive radio observations produced by $\sim 10 \mathrm{keV}$ electrons. Observers associated the GLEs with flares, but the radio observers provided the first evidence of two acceleration mechanisms and anticipated the importance of shock waves.

\subsection{The Spatial Distribution}

Parker (1963) not only described the continuous flow of the solar wind and the interplanetary magnetic field, he was aware of turbulence of that field and described the pitch-angle-scattering-induced diffusion of energetic particles flowing out along the field lines, an important description we still use often for ion transport along $\boldsymbol{B}$ (see Sect. 5.1.1). However, in time we learned that all SEP features, such as their extreme longitudinal spread, do not arise solely from diffusion. 


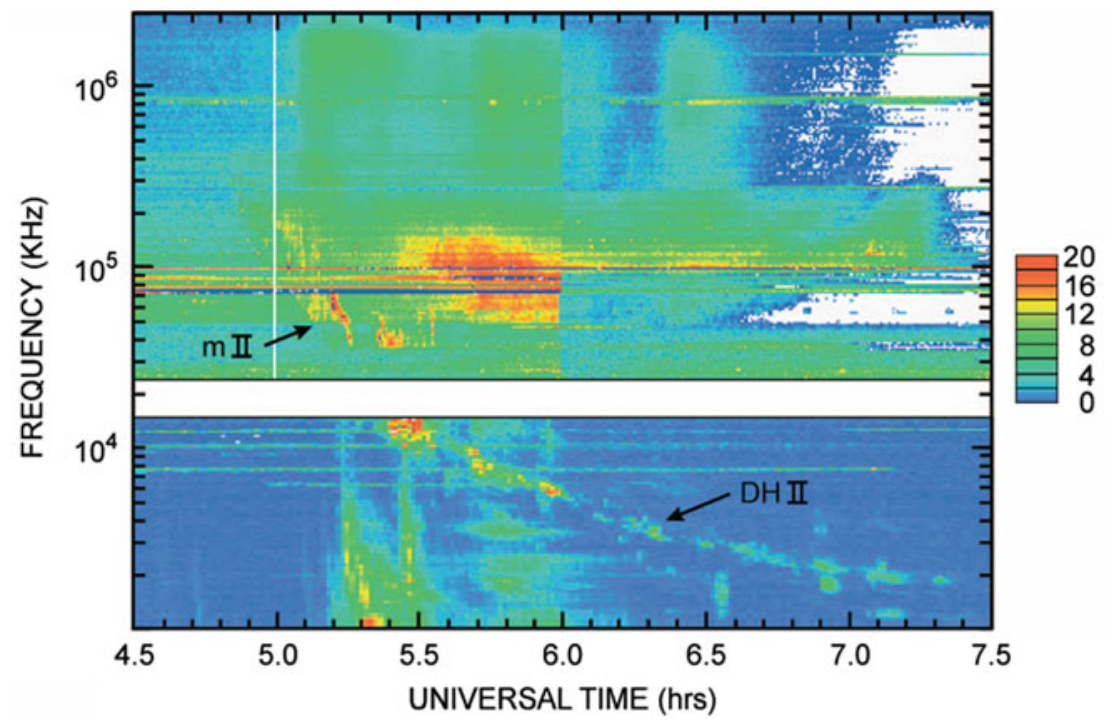

$\mathrm{dB}$

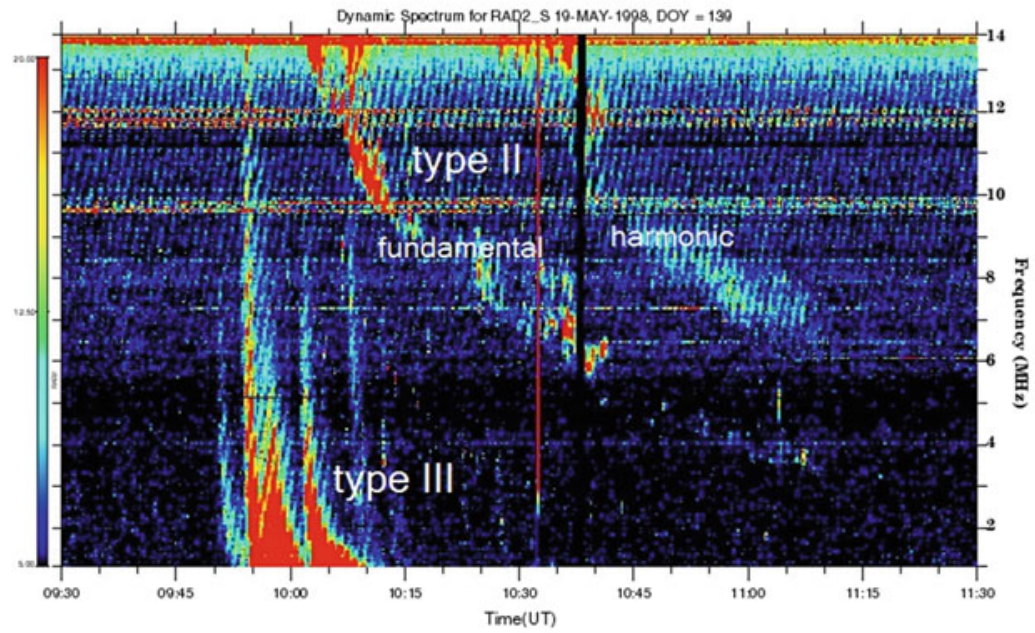

Fig. 2.1 The lower panel shows the dynamic radio spectrum obtained by the Wind/WAVES instrument in May 1998 with type II and type III bursts. The upper panel shows the type II burst associated with the large SEP event of 26 December 2001 as it propagates from the ground-based metric ( $\geq 25 \mathrm{MHz})$ Hiraiso station to the Wind/WAVES decametric-hectometric $\mathrm{DH}(\leq 14 \mathrm{MHz})$ regime (Cliver et al. 2004, (C) AAS)

\subsubsection{Lateral Diffusion and the Birdcage Model}

"A man with only a hammer treats every problem like a nail." In early studies of large SEP events all the distributions seemed like they must involve particle transport from a point-source flare, and diffusion theory was the transport tool of choice. The time dependence of the proton intensities had a smooth rise and a long, slow 
decay. Yet SEP events apparently associated with flares had such a wide span of solar longitudes, exceeding $180^{\circ}$. You could see the flares so they must be the source. Perhaps the particles from the flare diffused through the solar corona somehow and then out along the magnetic field lines toward Earth (Reid 1964).

In diffusion models, all of the physics of scattering is put into the diffusion coefficients, but when these coefficients are treated as adjustable parameters, their reality can become tenuous. Did the particles actually cross magnetic-field lines?

In fact, there was an early idea of a "fast propagation region" (Reinhard and Wibberenz 1974) of $\approx 60^{\circ}$ in solar longitude after which particles diffused away more slowly. The authors did consider that the "fast propagation region" might actually be the surface of a shock wave, yet could not believe it to be the actual source of the acceleration. Shock waves were generally well known in 1974.

In the birdcage model (Newkirk Jr. and Wenzel 1978), arcades of coronal loops formed structures like wires of a birdcage, spreading particles across the corona. At the footpoints of the loops the fields were somehow connected to the next series of loops, and so on across the Sun. Transport through this grid was simply assumed to be diffusive and these diffusive transport models held sway for decades.

\subsubsection{Large Scale Shock Acceleration and CMEs}

A direct challenge to the birdcage model came first from Mason et al. (1984). They observed the abundances of low-energy $\mathrm{H}, \mathrm{He}, \mathrm{C}, \mathrm{O}$, and Fe ions over an extended time as connection longitudes drifted far $\left(\sim 120^{\circ}\right)$ from the source. Relative abundances of these ions representing different magnetic rigidities were not altered by their alleged complex journey through the coronal birdcage. The authors suggested that the ions must actually result from large-scale shock acceleration (LSSA). Shocks can easily cross magnetic field lines, accelerating particles locally across a broad surface, wherever they go. LSSA also helped explain the long duration of the gradual events, especially at low energies, where the shocks continue acceleration as they come far out from the Sun.

In the same year Kahler et al. (1984) found a 96\% correlation between the largest energetic SEP events and fast, wide CMEs. This paper strengthened preliminary associations found during the earlier Skylab mission when CME observations began to become common.

It has long been known that particles are accelerated at shock waves. SEP intensities often peak as a shock wave passes, indicating a local source from which particles diverge (Sect. 5.4). These peaks were called "energetic storm particle" or "ESP events." However, it has seemed difficult to communicate that the particles that arrived earlier than the ESP peak came from that same shock that was much stronger when it was nearer the Sun (e.g. Reames et al. 1996). 


\subsubsection{The Longitude Distribution}

When larger numbers of gradual SEP events had been accumulated, it became possible to organize them as a function of their apparent solar source longitude. Even today with multiple spacecraft available it is difficult to study many individual events by observing each of them with multiple spacecraft at several convenientlyspaced longitudes. Cane et al. (1988) did the next best thing, studying 235 large events of $>20-\mathrm{MeV}$ protons observed on IMP and ISEE 3 by binning them as a function of their associated source longitudes. The authors concluded that the most important factor organizing the time profiles of large SEP events was the existence of an interplanetary-shock source and the curved Parker-spiral magnetic field which the particles were largely constrained to follow. Figure 2.2 shows a version of their findings.

In Fig. 2.2, the three cases shown are described as follows:

1. A spacecraft on the East flank of the shock (a western solar-source longitude) sees a fast intensity increase early, when it is magnetically well-connected to the strongest source at the "nose" of the shock as it first appears near the Sun. At later times the intensity decreases as the magnetic connection point moves gradually around the shock toward its weaker eastern flank. When this flank of the shock would be expected to pass the spacecraft, the shock may be very weak or may have dissipated completely so far around from the nose.

2. A spacecraft observing a source near central meridian is magnetically connected far to the West of the shock nose early in the event but the intensity increases as the shock moves outward and the connection point approaches the nose. The connection to the shock nose occurs as the shock itself passes the spacecraft. Thereafter, the intensity may decline suddenly as the spacecraft passes inside the CME driving the shock.

3. A spacecraft on the West flank of the shock (an eastern source on the Sun) is poorly connected to the source but its connection and the observed intensities improve with time, reaching a maximum behind the shock when it encounters field lines that connect it to the nose of the shock from behind.

We will see that later observations of individual events from multiple spacecraft generally supported the pattern seen in Fig. 2.2 (e.g. Fig. 5.16).

\subsubsection{Scatter-Free Events}

Does ambient turbulence in the interplanetary medium cause pitch-angle scattering of the particles flowing out from the Sun? The classic Fig. 2.3 from Mason et al. (1989) provides an interesting answer especially late in an event.

Mason et al. (1989) showed that most ${ }^{3} \mathrm{He}$-rich events (like that on 23 October 1978) actually propagate scatter free, i.e. with $\lambda \geq 1$ AU. We will see in Sect. 5.1.2 that in more intense events the streaming protons may be scattered early by 


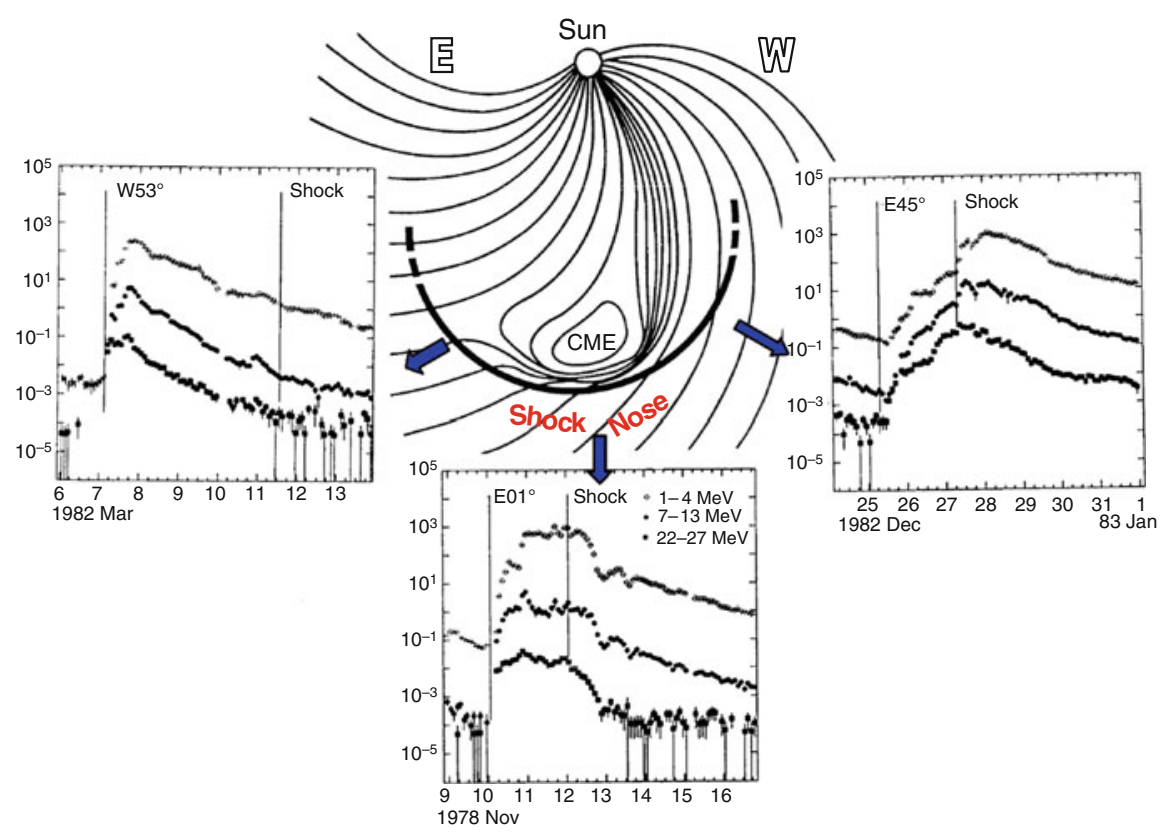

Fig. 2.2 Variation of the appearance of typical SEP events is shown as viewed from three solar longitudes (see text; after Reames 1999 (C) Springer; see also Cane et al. 1988, Reames et al. 1996)

self-amplified waves, but the slow decrease late in gradual events actually occurs when ions are adiabatically trapped in a magnetic reservoir (Sect. 5.7) behind the CME and shock. There is little scattering in the reservoir, but intensities decrease because the volume of the reservoir expands. Diffusion might be appropriate earlier in an event, but it does not produce the slow intensity decay of the large event, as the profile of the small scatter-free event on October 23 shows. Slow decays of SEPs are yet another misapplication of diffusion theory (see Sect. 5.7).

\subsubsection{Field-Line Random Walk}

While particles do not easily cross field lines, and the field lines may not join fortuitously, as suggested by the birdcage model, their footpoints do engage in a random walk which has the effect of spreading the longitude distribution of particles injected upon them (Jokipii and Parker 1969; Giaclaone and Jokipii 2012). The footpoints of the open field lines are imbedded in turbulent velocity fields that cause adjacent lines of force to execute a random walk relative to each other in time, as each stage of the evolving field pattern is carried out by the solar wind. Field lines are also buffeted by turbulence from the passage of CMEs. Thus, even at quiet times, field lines from any small region on the Sun have a distribution that is spread about the Parker spiral so that particles from a compact impulsive SEP event have a 


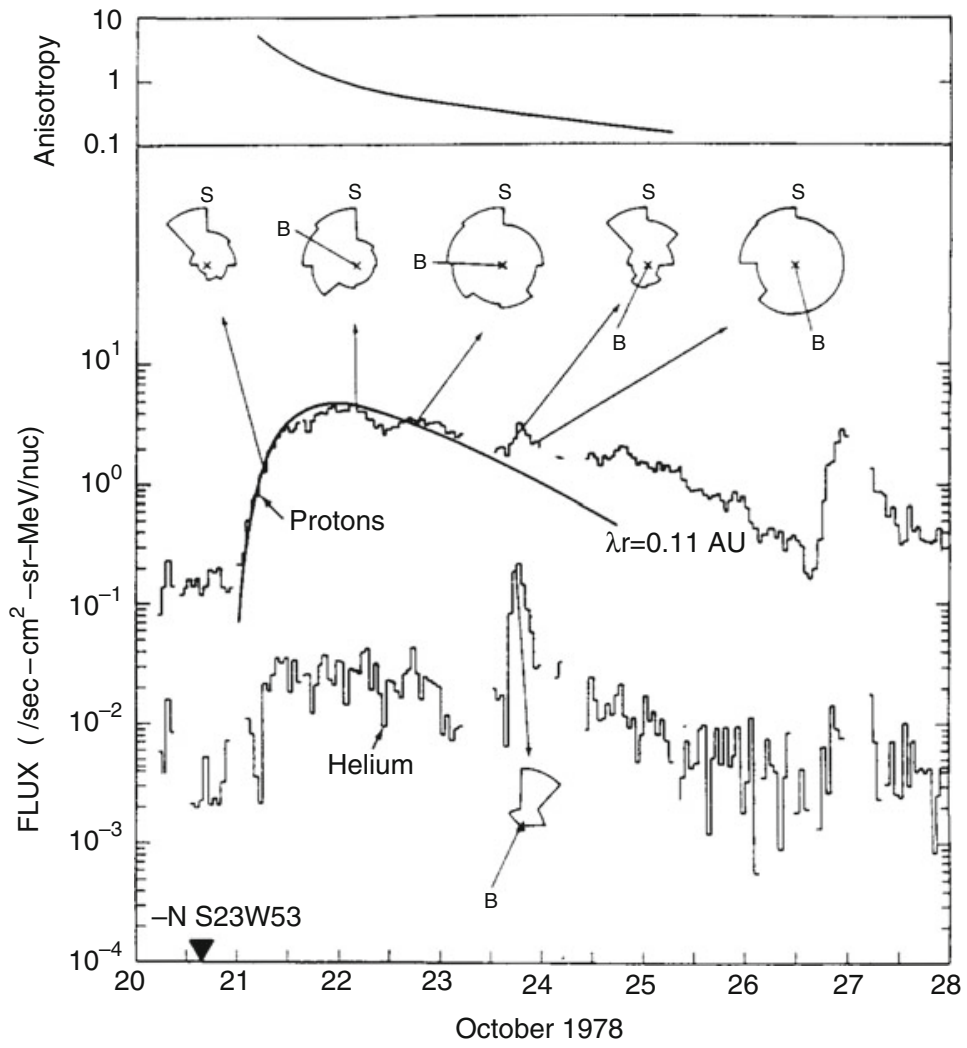

Fig. 2.3 Intensities and angular distributions of $\sim 1 \mathrm{MeV} \mathrm{amu}^{-1} \mathrm{H}$ and $\mathrm{He}$ are shown for a large SEP event of 21 October, 1978 and for the newly anisotropic flow from a small ${ }^{3} \mathrm{He}$-rich event on 23 October. A diffusion fit, to the proton intensity is shown with a radial component of the scattering mean free path of $0.11 \mathrm{AU}$. How can scattering spread particles in time so much in the large event, but barely scatter those from the small event in its wake (Mason et al. 1989, (C) AAS)? The long duration of the large event comes from continuing acceleration, then from trapping behind the CME, not from scattering

Gaussian-like longitude (and latitude) distribution. In Fig. 2.4, this contributes to the longitude spread of the impulsive events shown in the right panel. In the left panel, the gradual events are also spread in longitude by the spatial extent of the shockwave source. Recent more-sensitive instruments on STEREO see small, delayed echoes of impulsive events at longitudes $>60^{\circ}$ distant at reduced fluence (Wiedenbeck et al. 2013) vs. gradual events over nearly $360^{\circ}$ (e.g. Reames et al. 1996). The spread in impulsive SEPs includes variations in $V_{S W}$ and random walk of the field lines (Giaclaone and Jokipii 2012). 


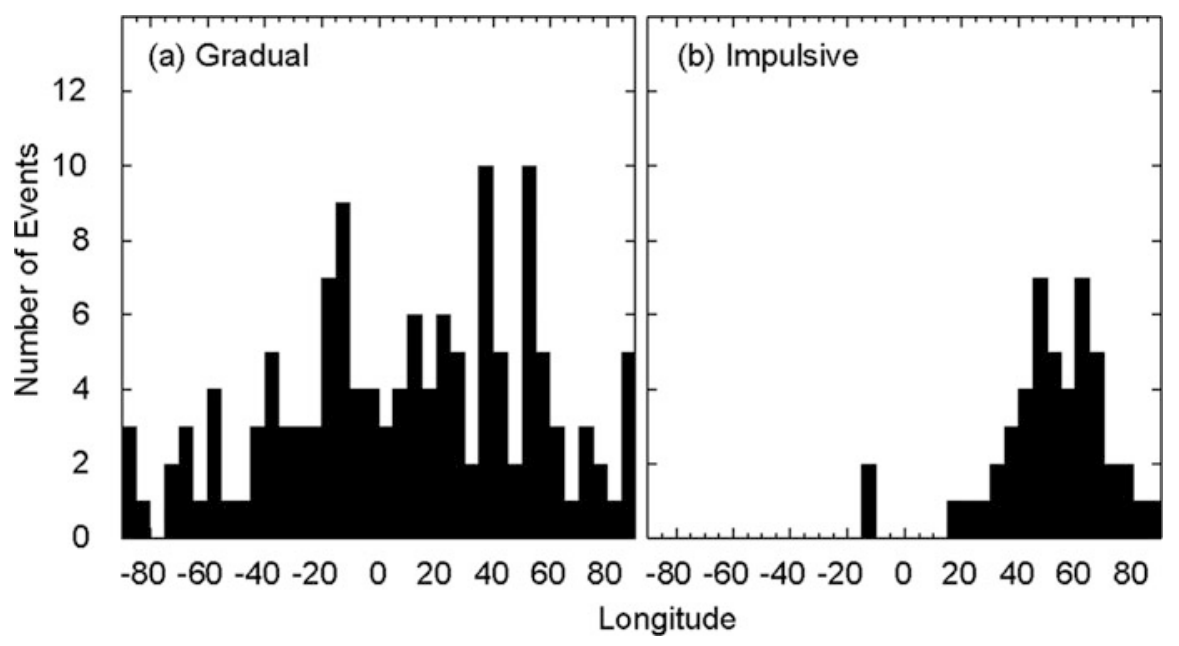

Fig. 2.4 Longitude distributions are shown for gradual SEPs (left) spread mainly because of the width of the shock source, and impulsive (right) SEP events spread by random walk of field lines and by variations in solar wind speed (Reames 1999 (C) Springer)

\subsection{Shock Theory}

Shock acceleration theory had an extensive history in GCR acceleration prior to its application to SEPs and that will not be repeated here. The plasma physics of shocks and shock acceleration has been reviewed by Jones and Ellison (1991; see also Lee 2005, Sandroos and Vainio 2007, Zank et al. 2007; Verkhoglyadova et al. 2015). Diffusive shock acceleration (DSA) occurs as ions are pitch-angle scattered back and forth across a shock wave, gaining an increment of velocity on each round trip. For an oblique shock wave, particles can gain additional energy in the $\boldsymbol{V}_{\mathbf{S}} \times \boldsymbol{B}$ electric field of the shock (e.g. Decker 1983).

Acceleration of a particle at a quasi-parallel shock may be considered approximately as a series of frame transformations which are randomly required as a particle scatters from upstream to downstream to upstream of the shock. On each round trip the particle has gained a velocity related to the velocity difference between the upstream and downstream scattering centers; in this difference, the shock speed can be augmented by the Alfvén speed of the four possible wave modes-inward and outward along $\boldsymbol{B}$ times two circular polarizations. The diffusion, the scattering against resonant waves, is determined by the particle's rigidity, but it gains in velocity. The rate of acceleration increases as the scattering mean free path decreases reducing the mean time between crossings. As accelerated particles stream away from the shock, they amplify resonant Alfvén waves of wave number $k \approx B / \mu P$, according to quasi-linear theory, where $P$ is the particle rigidity and $\mu$ its pitch-angle cosine. These amplified waves increase the resonant scattering of the ions that follow behind (see Sect. 5.1.2). Assuming $\mu \approx 1$ for simplicity, this traps particles of $P$ near 
the shock, increasing their energy to a higher value, say rigidity $P_{2}$, where they stream out, amplifying waves that resonate with $P_{2}$ which are trapped and accelerated to $P_{3}$, etc. Continuing this process indefinitely can lead to a power-law spectrum where the power depends upon the shock compression ratio. However, SEP spectra are produced by CME-driven shocks with finite lives and diminishing strength so they have spectral breaks or "knees" where spectra steepen, generally above $10 \mathrm{MeV}$ (see Fig. 3.7), and produce complex behavior like that we will see in Figs. 2.9 and 2.10. The equations that control particle transport, scattering, and wave growth will be presented in Sects. 5.1.2 and 5.1.3.

Quasi-linear theory actually assumes that the energy density in wave turbulence is small with respect to the energy density in the field, $\delta B / B<<1$, a condition that is most likely violated at strong shocks which approach or even exceed the "Bohm limit" where the proton scattering mean free path equals its gyroradius. Lee (1983) applied equilibrium DSA theory to explain proton acceleration at interplanetary shocks assuming $\mu \approx 1$. At equilibrium, the growth of upstream waves that resonate with each rigidity is just sufficient to replace the waves being swept into the shock. In contrast, Zank et al. (2000) found that shock acceleration could produce $\mathrm{GeV}$ protons near the Sun, assuming that turbulence reaches the Bohm limit $\delta B /$ $B \approx 1$ at the shock.

$\mathrm{Ng}$ et al. (2003) considered the self-consistent, time-dependent particle transport with amplification of Alfvén waves, and $\mathrm{Ng}$ and Reames (2008) extended the calculation to the time-dependent shock acceleration of protons to energies of $>300 \mathrm{MeV}$.

It is not uncommon to consider the action of two or more shocks on a population of particles (e.g. Gopalswamy et al. 2002). The equilibrium energy spectrum for two consecutive shocks is derived as Eq. (5.9) (Sect. 5.4) and is a power-law spectrum with the power dominated by the compression ratio of the strongest shock. It is appropriate to assume that the shocks contribute sequentially; pre-acceleration of seed ions may increase the probability of secondary acceleration. However, there is no stronger collaborative effect; shock acceleration occurs within a modest number of proton gyroradii of the shock and CME-driven shocks crossing each other spend a negligible time at such a small separation.

\subsection{Element Abundances}

The earliest observations of heavier elements in SEP events were made using nuclear-emulsion detectors on sounding rockets launched into large SEP events. Fichtel and Guss (1961) observed C, N, and O nuclei above $25 \mathrm{MeV} \mathrm{amu}^{-1}$. The observations were extended to $\mathrm{Fe}$ by Bertsch et al. (1969). For the early measurements, the presence of SEPs was detected by a riometer, which measures radio absorption produced by ionization of the polar cap region produced by high intensities of SEPs. The riometer was used as an indication to fire sounding rockets above the atmosphere to measure SEP abundances from Ft. Churchill in northern Manitoba, Canada. 


\subsubsection{First lonization Potential (FIP) and Powers of A/Q}

Improving measurements led to comparison of element abundances in SEP events with those in the solar photosphere and corona (e.g. Webber 1975; Webber et al. 1975; Cook et al. 1984). The measurements were summarized in the review of Meyer (1985). He found two factors that influenced element abundances in large SEP events $\left({ }^{3} \mathrm{He}\right.$-rich events were excluded). There was one component, present in all events that depended upon the first ionization potential (FIP) of the elements, and a second variable component that he called "mass bias" actually depending upon the mass-to-charge ratio $A / Q$ of the ions. The $A / Q$ dependence differed with time and from one event to another. The FIP dependence that was shown in Fig. 1.6 represents average abundances at the coronal origin of SEPs, relative to the corresponding photospheric abundances. Elements with FIP above about $10 \mathrm{eV}$ are neutral atoms in the photosphere while lower-FIP elements are ionized. The ions are more rapidly swept up into the corona, as by Alfvén waves (e.g. Laming 2004, 2009) and thus have higher relative abundances there.

An increasing or decreasing power-law dependence on the $A / Q$ ratio of the ions was clearly found by Breneman and Stone (1985) and is shown in the left panel of Fig. 2.5. Breneman and Stone (1985) used the newly available ionization-state measurements of Luhn et al. (1984) to determine $Q$.

After languishing for over 30 years, these power-laws have gained renewed interest. The pattern of ionization states $Q$ depends upon the plasma temperature (see Fig. 5.11) and it has recently been shown (Reames 2016) that the pattern of the grouping of elements in enhancement vs. $A / Q$ (Fig. 2.5, right panel) determine the
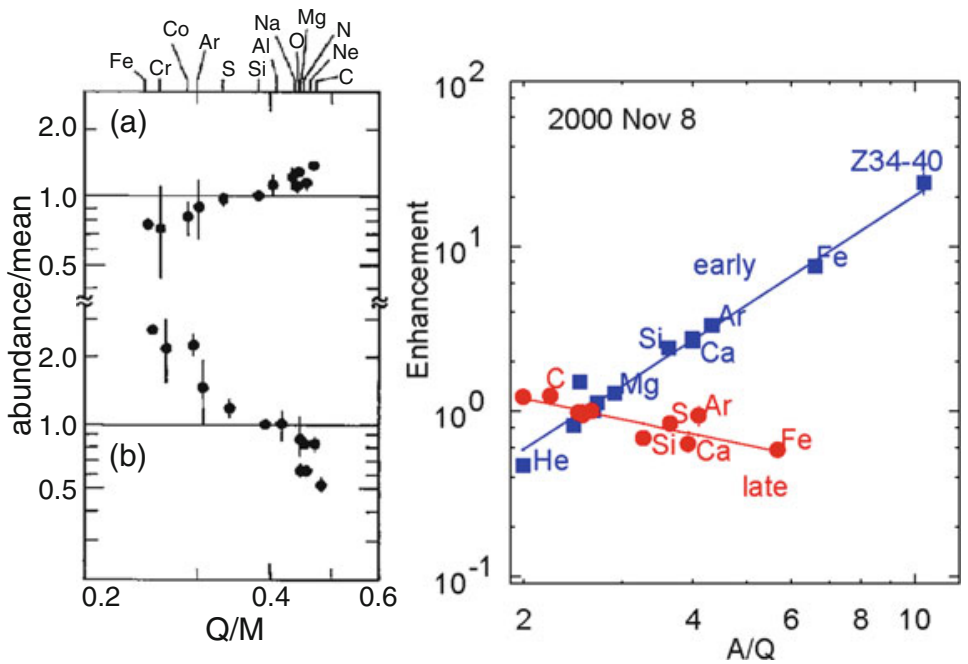

Fig. 2.5 The left panel shows the dependence of elemental abundances on the charge-to-mass ratio $Q / M$ (our $Q / A$ ) for two large SEP events (a and b) by Breneman and Stone (1985 C) AAS). The right panel shows the $A / Q$ dependence early (blue) and late (red) in a large SEP event (Reames 2016; see Fig. 5.13 (C) Springer) 
source-plasma temperature (see Sect. 5.6). In fact, grouping of elements $\mathrm{C}-\mathrm{Mg}$ with similar enhancements and $A / Q$ in the left panel of Fig. 2.5 suggests a temperature of about 1.5 MK, those in the right panel are both $1.0 \mathrm{MK}$. But we are getting ahead of our story (see Sect. 5.6).

\subsection{2 ${ }^{3}$ He-rich Events}

The first observation of ${ }^{3} \mathrm{He} /{ }^{4} \mathrm{He}$ in SEP events (Hsieh and Simpson 1970) showed some evidence of enhancement which aroused interest because of the possibility that ${ }^{3} \mathrm{He}$ could be produced in nuclear reactions in flares, but not when Serlemitsos and Balasubrahmanyan (1975) found ${ }^{3} \mathrm{He} /{ }^{4} \mathrm{He}=1.52 \pm 0.10$ but ${ }^{3} \mathrm{He} /{ }^{2} \mathrm{H}>300$. With no evidence of other reaction products, like ${ }^{2} \mathrm{H}$ or ${ }^{3} \mathrm{H}$, it became clear that a new acceleration process was involved, since ${ }^{3} \mathrm{He} /{ }^{4} \mathrm{He} \approx 5 \times 10^{-4}$ in the solar wind. It also became apparent that there were other abundance enhancements, such as $\mathrm{Fe} / \mathrm{O}$ that was $\sim 10$ times larger than in the solar wind (e.g. Gloeckler et al. 1975). However, there is still no evidence of nuclear-reaction secondaries, ${ }^{2} \mathrm{H},{ }^{3} \mathrm{H}, \mathrm{Li}$, $\mathrm{Be}, \mathrm{B}$, etc. in the SEPs; $\gamma$-ray and neutron measurements tell us they are produced in flare loops (Sect. 4.9), but are magnetically trapped there and cannot get out.

The next generation of measurements of ${ }^{3} \mathrm{He}$-rich events (Fig. 2.6) led to their association with non-relativistic electron events (Reames et al. 1985) and with type III radio bursts (Reames and Stone 1986). Thus Lin's (1970) "pure" electron events were actually ${ }^{3} \mathrm{He}$-rich or "impulsive" SEP events and were associated with the type III-burst electron events discussed by Wild et al. (1963) that we saw in Sect. 2.2. While these events were also Fe-rich, Fe/O was not correlated with ${ }^{3} \mathrm{He} /{ }^{4} \mathrm{He}$ (e.g. Mason et al. 1986), opening the possibility and the need for two different enhancement mechanisms.

The unique ${ }^{3} \mathrm{He}$ enhancement suggested a resonant interaction with plasma waves. The earliest mechanism suggested was based upon the selective heating by absorption of ion-sound waves (Ibragimov and Kocharov 1977; Kocharov and Kocharov 1978, 1984). However, Weatherall (1984) found that this mechanism did not have the sensitivity to ion charge needed to account for the observed abundances. Fisk (1978) and Varvoglis and Papadopoulis (1983) suggested selective heating of ${ }^{3} \mathrm{He}$ by absorption of electrostatic ion cyclotron waves at the ${ }^{3} \mathrm{He}$ gyrofrequency; Winglee (1989) also invoked ion-ion streaming instability in an effort to enhance heavy ions. Riyopoulos (1991) considered electrostatic two-ion $\left(\mathrm{H}-{ }^{4} \mathrm{He}\right)$ hybrid waves. Some of the mechanisms suggested during this period required high values of ${ }^{4} \mathrm{He} / \mathrm{H}$ in the source plasma, most required a second unspecified physical process for preferential acceleration of the pre-heated ions from the thermal distribution, such as a shock wave.

Temerin and Roth (1992) and Roth and Temerin (1997) found that the streaming electrons that produce the type III bursts would generate electromagnetic ion cyclotron (EMIC) waves near the gyrofrequency of ${ }^{3} \mathrm{He}$. Ions mirroring in the converging magnetic field could be accelerated as they continue to absorb the waves, in analogy with the "ion conics" seen in the Earth's aurora. The strength of this model, with 

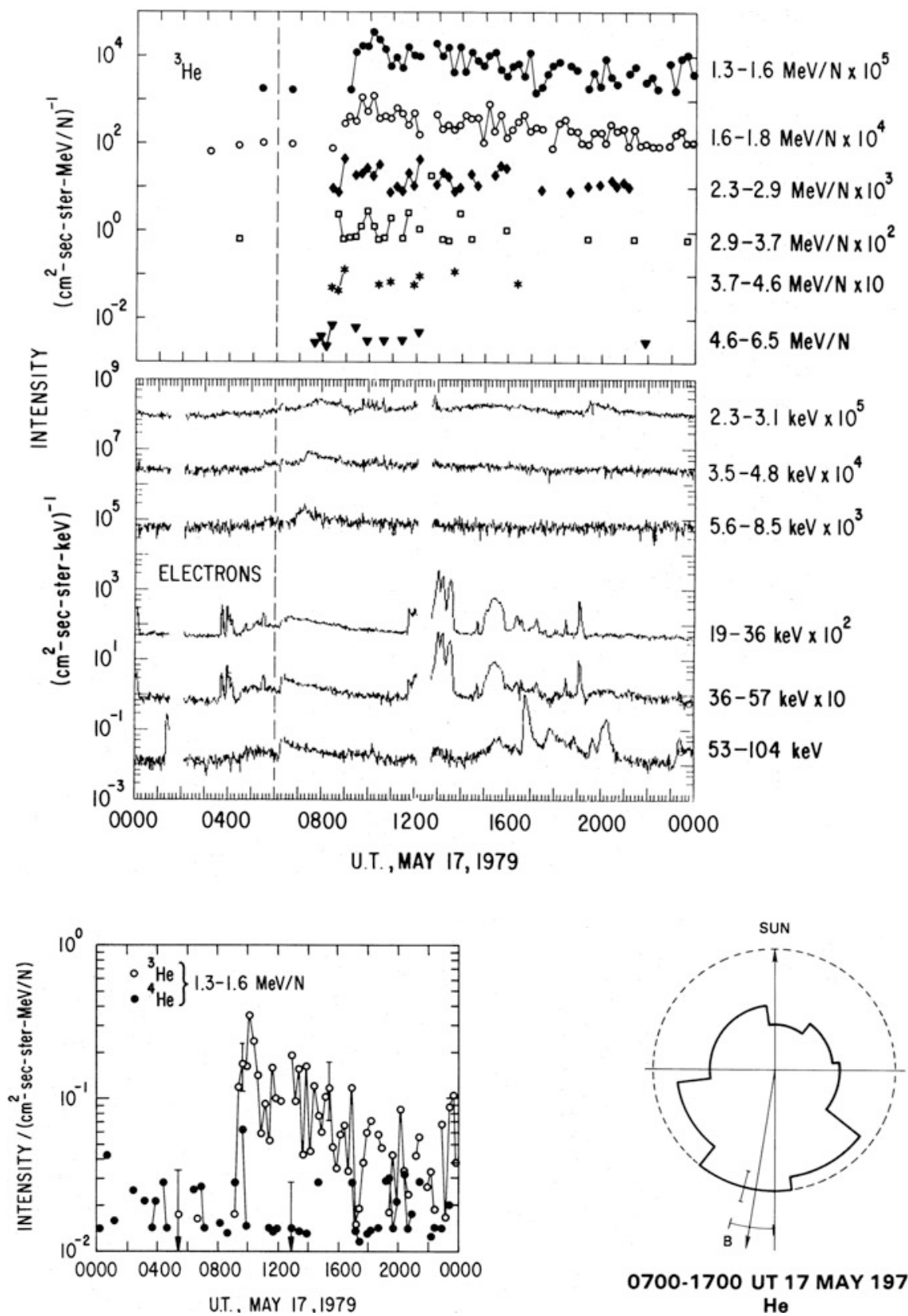

0700-1700 UT 17 MAY 1979 $\mathrm{He}$

Fig. 2.6 Intensities of ${ }^{3} \mathrm{He}$ and electrons of various energies (upper panels) show velocity dispersion (i.e. fastest particles arrive first after traveling $\sim 1$ AU from the Sun) while intensities of ${ }^{3} \mathrm{He}$ and ${ }^{4} \mathrm{He}$ (lower left) show dominance of ${ }^{3} \mathrm{He}$, with ${ }^{4} \mathrm{He}$ only at background, and the angular distribution (lower right) shows outward flow of ${ }^{3} \mathrm{He}$ along the magnetic field $\mathbf{B}$, all in a small ${ }^{3} \mathrm{He}-$ rich SEP event of 17 May 1979 (Reames et al. 1985, (C) AAS) 
more robust waves, was that (1) it explained and used the strong association observed between type-III electrons and ${ }^{3} \mathrm{He}$-rich events, (2) it produced ${ }^{3} \mathrm{He}$ acceleration, not just preheating, and (3) the streaming electrons provided a selfconsistent source for the resonant waves that preferentially energized ${ }^{3} \mathrm{He}$, although Litvinenko (1996) also considered EMIC waves. The authors suggested that heavier ions were accelerated through resonance with the second harmonic of their gyrofrequencies, but this required specific ionization states and did not produce the extreme and uniform increase in enhancement of the heavy elements with $Z>50$ that was observed subsequently (e.g. Reames 2000; see also Reames et al. 2014a, b), as we shall see in Chap. 4. Miller et al. (1993a, b) considered electron beam generation of other wave modes such as sheer-Alfvén waves and their effect on heavy ions, and Steinacker et al. (1997) considered effects of broadened spectral lines produced by thermal damping in a hot (2.4-4.5 MK) plasma, producing the broadened "He valley" of damping which controls the wave regions left available for absorption and enhancement of various heavier ions.

Ho et al. (2005) found that there was an upper limit to the fluence of ${ }^{3} \mathrm{He}$ in events so that increasingly large impulsive events had decreasing ${ }^{3} \mathrm{He} /{ }^{4} \mathrm{He}$ ratios. This agreed with an estimate by Reames (1999) that an impulsive event can accelerate and deplete most of the ${ }^{3} \mathrm{He}$ in a typical flare (or jet) volume.

${ }^{3} \mathrm{He}$-rich events were traced to their solar sources by Nitta et al. (2006) and by Wang et al. (2006) and there was a growing association with narrow CMEs that has become a clear association with solar jets (Kahler et al. 2001; Bučík et al. 2018; Bučík 2020; see also Reames et al. 2014a).

\subsubsection{The Seed Population for Shocks}

For a time, it seemed that impulsive and gradual events might be distinguished by their element abundances alone. Impulsive events were ${ }^{3} \mathrm{He}$-rich, weren't they? Then Mason et al. (1999) found enhancements of ${ }^{3} \mathrm{He}$ in large SEP events that clearly should otherwise be called gradual. In fact, there were even large ${ }^{3} \mathrm{He}$ enhancements during relatively quiet times. Earlier evidence of this had been seen by Richardson et al. (1990). The mass distribution in Fig. 2.7 clearly shows ${ }^{3} \mathrm{He}$, and although the amount is small, it is 5 times the solar-wind abundance. The authors suggested that the ${ }^{3} \mathrm{He}$, and also Fe, are suprathermal remnants of previous impulsive SEP events. These impulsive-suprathermal ions contribute to the seed population for subsequent shock acceleration (see Tylka et al. 2001).

Exploring the seed population, Desai et al. (2001) found ${ }^{3} \mathrm{He}$ intensity increases at shocks in 25 SEP events with enhancements of 3 to 600 relative to the solar wind ratio, and Desai et al. (2003) found Fe/O at the shock was correlated with $\mathrm{Fe} / \mathrm{O}$ upstream. Figure 2.8 shows intensities of ${ }^{3} \mathrm{He},{ }^{4} \mathrm{He}, \mathrm{O}$, and $\mathrm{Fe}$ before and during a strong shock event. The quiet period labeled A, "upstream" is both ${ }^{3} \mathrm{He}$-rich and has 
Fig. 2.7 The mass distribution of He is shown directly (solid - left scale) and with an expanded scale (open - right scale) to show ${ }^{3} \mathrm{He}$ at $0.2-2.0 \mathrm{MeV} \mathrm{amu}^{-1}$ during quiet times (Mason et al. 1999, (C) AAS)

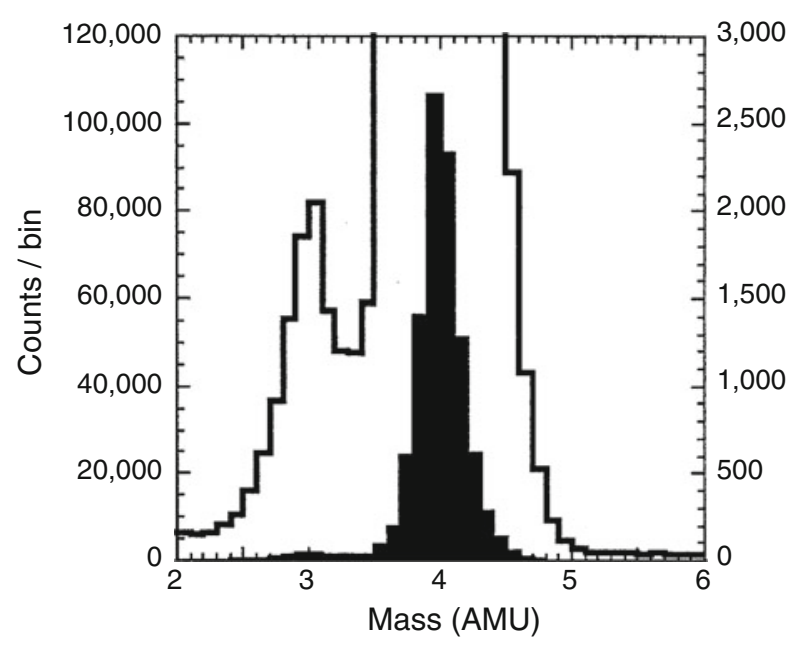

$\mathrm{Fe} / \mathrm{O}>1$ while the later period B on 24 June is just extremely Fe-rich. These strong $\mathrm{Fe} / \mathrm{O}$ enhancements do not persist at the shock, but there clearly must be ${ }^{3} \mathrm{He}$ in the seed population, suggesting that it contains suprathermal ions from earlier impulsive SEP events. The correlation of Fe/O at the shock with that upstream is consistent with that interpretation. Note, however, that most of the ions at this shock peak $d o$ not come from ${ }^{3} \mathrm{He}$-rich impulsive suprathermals. We will see that these ESP peaks represent ambient coronal material in most cases, although suprathermal ions may also contribute.

Tylka et al. (2005) found that in two otherwise-similar, large SEP events, the energy dependence of $\mathrm{Fe} / \mathrm{C}$ above $\sim 10 \mathrm{MeV}$ amu $^{-1}$ suddenly increased in one event and decreased in the other, as shown in the left panel of Fig. 2.9. The authors considered the possible selection effect of impulsive suprathermal ions caused by differences in shock geometry. In quasi-perpendicular shock waves, with $\boldsymbol{B}$ perpendicular to the shock normal, injected ions may need a higher speed to reacquire the shock from downstream, so that pre-accelerated impulsive suprathermal ions would be preferentially selected as shown in the right panel of Fig. 2.9. Tylka and Lee (2006) calculated the effect different seed populations and shock geometries could have on the energy dependence of Fe/C. The higher-energy effects occur because the location of the high-energy "knee" (Sect. 3.4) where the power-law shock spectra roll downward, depends upon $Q / A$ of the ions and $\sec \theta_{\mathrm{Bn}}$, the angle between $\boldsymbol{B}$ and the shock normal. Coronal- and impulsive-suprathermal ions have different values of $Q$ and thus contribute differently above the spectral knee.

Tylka and Lee (2006) assumed that the shock spectrum of species $i$ varied as $j_{i}(E)=k_{i} E^{-\gamma} \exp .\left(-E / E_{0 \mathrm{i}}\right)$, a form originally suggested by Ellison and Ramaty (1985). Then letting $E_{0 \mathrm{i}}=E_{0} \times\left(Q_{\mathrm{i}} / A_{\mathrm{i}}\right) \times\left(\sec \theta_{\mathrm{Bn}}\right)^{2 /(2-\gamma)}$, where $E_{0}$ is the proton knee energy, a wide variety of energy dependence of $\mathrm{Fe} / \mathrm{O}$ may be seen as in Fig. 2.10. 


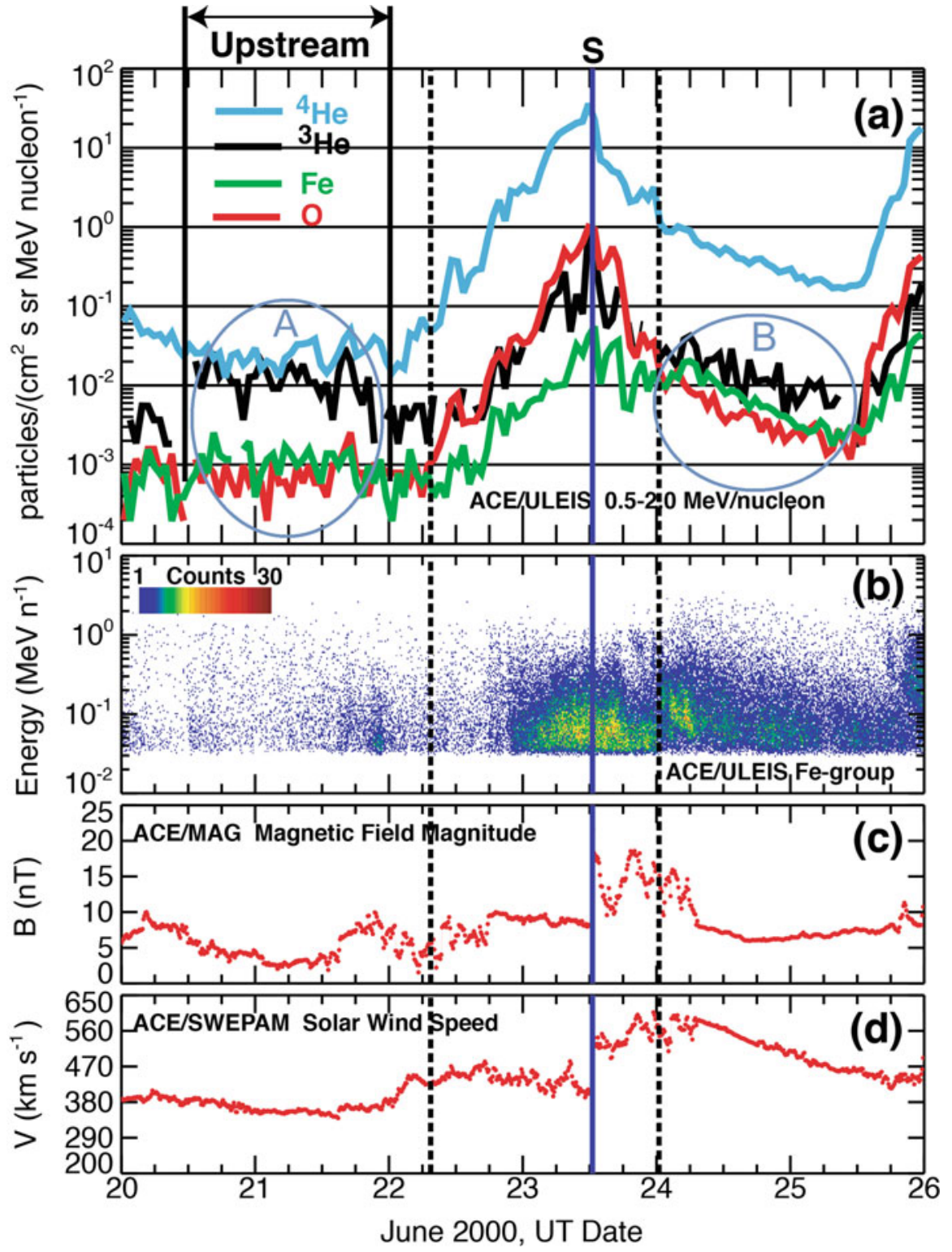

Fig. 2.8 (a) Intensities of $0.5-2.0 \mathrm{MeV} \mathrm{amu}{ }^{-1}{ }^{3} \mathrm{He},{ }^{4} \mathrm{He}, \mathrm{O}$ and $\mathrm{Fe}$ are shown during a large SEP event, with (b) a histogram of $\mathrm{Fe}$ arrivals, (c) the magnetic field $\mathrm{B}$, and (d) the solar wind speed. ${ }^{3} \mathrm{He}$ is clearly accelerated, peaking at the shock, $\mathrm{S}$, but is not as strongly enhanced as in the ${ }^{3} \mathrm{He}$-rich period labeled A, "upstream." In quiet period $\mathbf{A}$ both ${ }^{3} \mathrm{He} /{ }^{4} \mathrm{He}$ and $\mathrm{Fe} / \mathrm{O}$ are enhanced; in $\mathbf{B}$ only $\mathrm{Fe} / \mathrm{O}$ is enhanced (Desai et al. 2003, (C) AAS). Quiet periods are frequently ${ }^{3} \mathrm{He}$ - and Fe-rich (Bučík et al. 2014, 2015; Chen et al. 2015) 

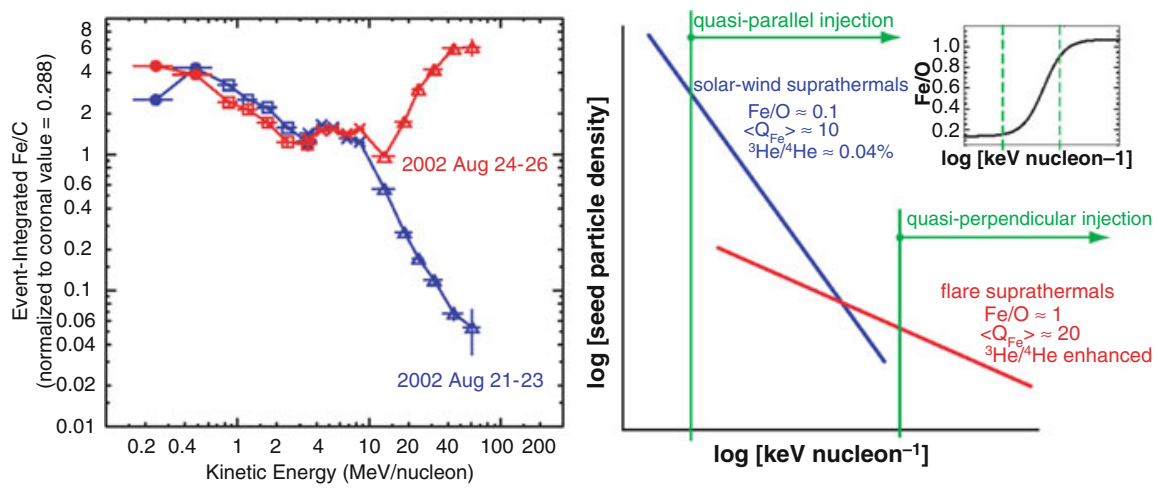

Fig. 2.9 The left panel compares the energy dependence of $\mathrm{Fe} / \mathrm{C}$ for two gradual events that are otherwise similar in their properties (Tylka et al. 2005 (C) AAS). The right panel shows hypothetical spectra of two sources of suprathermal ions where different injection thresholds will yield different abundance ratios (Tylka et al. 2005). Clearly, it would be unwise to use measurement of $\mathrm{Fe} / \mathrm{C}$ above $\sim 10 \mathrm{MeV} \mathrm{amu}{ }^{-1}$ in an attempt to distinguish impulsive and gradual SEP events

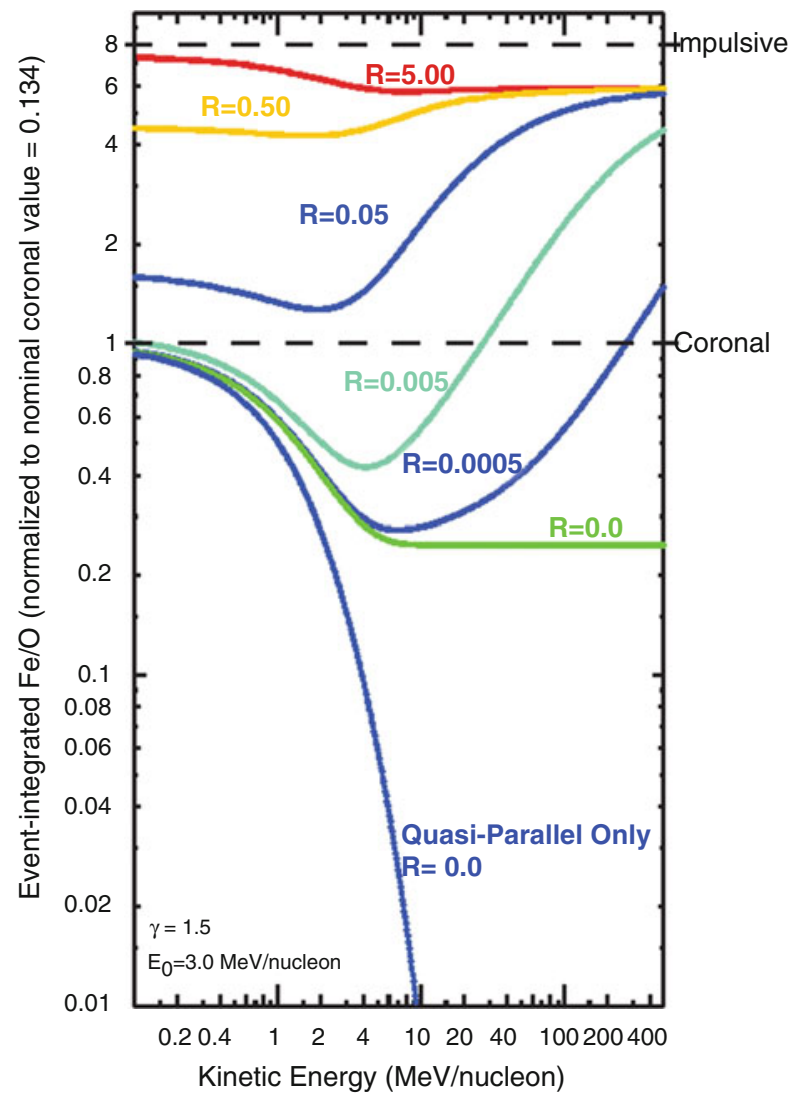

Fig. 2.10 The energy dependence of $\mathrm{Fe} / \mathrm{O}$ is shown as a function of $R$ which is the ratio, in the seed population, of $\mathrm{O}$ in impulsive suprathermal ions to coronal ions. The values of $\gamma$ and $E_{0}$ assumed for this case are shown in the lower left corner of the figure (Tylka and Lee 2006 (C) AAS) 
In fact, the seed population for shock acceleration can consist of ambient coronal material as well as residual suprathermal ions from previous impulsive and gradual SEP events. However, Giacalone (2005) noted that high turbulence near the shock with $\delta B / B \approx 1$ would allow oblique shocks better access to the low-energy seed population and diminish the selective dependence on $\theta_{\mathrm{Bn}}$.

For further studies of the dependence of the spectral break, of its power-law dependence upon $Q / A$ and the variation with shock geometry, see Li et al. (2009) and Zhao et al. (2016). The latter authors conclude that the energy of the spectral break depends upon $(Q / A)^{\delta}$ where $0.4<\delta<1.3$, with break energies varying between 10 and $120 \mathrm{MeV} \mathrm{amu}{ }^{-1}$. Note that abundances such as $\mathrm{Fe} / \mathrm{O}$ will become affected when $\mathrm{Fe}$ is above its break but $\mathrm{O}$ is not.

\subsection{Ionization States}

Some of the earliest direct measures of SEP ionization states were the direct measurements at $0.34-1.8 \mathrm{MeV} \mathrm{amu}{ }^{-1}$ for $\mathrm{Fe}$ (Luhn et al. 1984, 1987). They found an average of $Q_{\mathrm{Fe}}=14.2 \pm 0.2$ for gradual events, corresponding to a plasma temperature of $\sim 2 \mathrm{MK}$, but a much higher value of $Q_{\mathrm{Fe}}=20.5 \pm 1.2$ for ${ }^{3} \mathrm{He}$-rich events. Either the ${ }^{3} \mathrm{He}$-rich events are much hotter, $10 \mathrm{MK}$, or, as we now believe, the ions may be stripped in transit away from the impulsive sources which lie a little deeper in the corona. Subsequently Leske et al. (1995) used geomagnetic cutoffs to find the $Q_{\mathrm{Fe}}=15.2 \pm 0.7$ at $15-70 \mathrm{MeV} \mathrm{amu}{ }^{-1}$ in large events and Tylka et al. (1995) found $Q_{\mathrm{Fe}}=14.1 \pm 1.4$ at $200-600 \mathrm{MeV} \mathrm{amu}^{-1}$.

More recently, DiFabio et al. (2008) found that the ionization states in impulsive SEP events increased with energy, suggesting that the ions had passed through enough material that electron stripping and capture were in equilibrium at each ion velocity. The authors suggested that the ions in impulsive events were accelerated below 1.5 $R_{S}$ where densities were higher, beginning at a temperature of 1-3 MK. It was once suggested that ${ }^{3} \mathrm{He}$-rich SEP events come from "high coronal flares", based upon their electron spectra, but the stripping of Fe associates them with the deepest known SEP sources. We will see in Sect. 3.1 that acceleration in gradual events does begin higher in the corona, at $2-3 \mathrm{R}_{\mathrm{S}}$.

A different approach to determining ionization states in impulsive events was taken by Reames et al. (1994). They noted that in average impulsive SEP events, the elements ${ }^{4} \mathrm{He}, \mathrm{C}, \mathrm{N}$, and $\mathrm{O}$ showed no enhancement relative to reference coronal abundances, $\mathrm{Ne}, \mathrm{Mg}$, and $\mathrm{Si}$ were enhanced by a factor of $\sim 2.5$, and Fe by a factor of $\sim 7$. This suggested that, at the time of acceleration, $\mathrm{C}, \mathrm{N}$, and $\mathrm{O}$ were fully ionized like $\mathrm{He}$, but that $\mathrm{Ne}, \mathrm{Mg}$, and $\mathrm{Si}$ were probably in a stable closed shell configuration with two orbital electrons. They suggested that this occurs in a temperature range of 3-5 MK. At higher temperatures, Ne would become stripped, have $Q / A=0.5$ like lighter elements, and could not be enhanced relative to them. At lower temperatures, $O$ could capture electrons and would no longer have $Q / A \approx 0.5$. More recent studies (Reames et al. 2014a, b) have lowered this range to 2-4 MK to account for (1) more accurate measurements that showed $\mathrm{Ne}$ enhancements exceeding those of $\mathrm{Mg}$, and 
$\mathrm{Si}$, (2) $\mathrm{O}$ enhancements causing decreased $\mathrm{He} / \mathrm{O}$ and $\mathrm{C} / \mathrm{O}$, and (3) a power-law fit in $A / Q$ extending to $(Z>50) / \mathrm{O}$ (see Sect. 4.6). These values of $2-4 \mathrm{MK}$ correspond to ambient electron temperatures in solar active regions where flares and jets occur. Thus we began to use abundances to measure temperatures.

The strong $A / Q$ dependence of the enhancements extending to a factor of $\sim 1000$ for (76 $\leq Z \leq 82) / O$ (e.g. Reames et al. 2014a, b) recently has been theoretically understood as occurring in collapsing islands of magnetic reconnection (e.g. Drake et al. 2009). These particle-in-cell simulations show that ions are Fermi-accelerated as they are reflected back and forth from the ends of the collapsing islands of magnetic reconnection (see Sect. 4.7).

While impulsive SEPs may have passed through the extremely small amount of matter required to attain equilibrium values of $Q$, they cannot have passed through enough material to lose significant energy, since the $Q^{2} / A$ dependence of the energy loss would destroy the strong $~ 1000$-fold enhancement observed for heavy elements such as $(76 \leq Z \leq 82) / O$.

Recent studies of the $A / Q$ dependence in gradual SEP events (Reames 2016) have found that most of these events $(69 \%)$ have source-plasma temperatures $\leq 1.6$ MK, consistent with shock acceleration of ambient coronal plasma (see Sect. 5.6). Only $24 \%$ of the events have active-region temperatures of 2.5-3.2 MK and thus include dominant enhancements from impulsive suprathermal seed ions.

Using the $A / Q$-dependence of abundance enhancements, with $Q$ vs. $T$ from atomic physics, these studies provide a new method of determining ionization states at the point of acceleration. This circumvents the effects of stripping that may be present in the ionization states measured later at $1 \mathrm{AU}$.

\subsection{Disappearing-Filament Events}

A "disappearing" filament occurs when a filament, which may have been visible in the corona for days, is suddenly destabilized and erupts within a CME, disappearing from its former position. An $\mathrm{H} \alpha$ brightening may form a classic double-ribbon pattern along the filament channel with slight heating and soft X-ray emission, but no hard X-ray emission or flaring occurs. Such events can produce a fast CME, a shock wave, and a substantial gradual SEP event, without the need of a flare or even a solar active region.

An early association of SEPs with filament changes was made by Sanahuja et al. (1983) but a clear example was the SEP event of 5 December 1981, shown in Fig. 2.11, identified and discussed by Kahler et al. (1986). Cane et al. (1986) found six other disappearing-filament-associated SEP events with a CME and shock but no impulsive phase or flare, and Gopalswamy et al. (2015) have extended this study to recent large gradual SEP events. They conclude that fast CMEs that produce GLEs attain a high speed at 2 or $3 R_{s}$ while those in filament eruptions begin slowly and 
Fig. 2.11 Intensities vs. time are shown for the disappearing-filamentassociated SEP event of 5 December 1981. The peak in the low energy protons on 8 December occurs at the time of shock passage at $1 \mathrm{AU}$

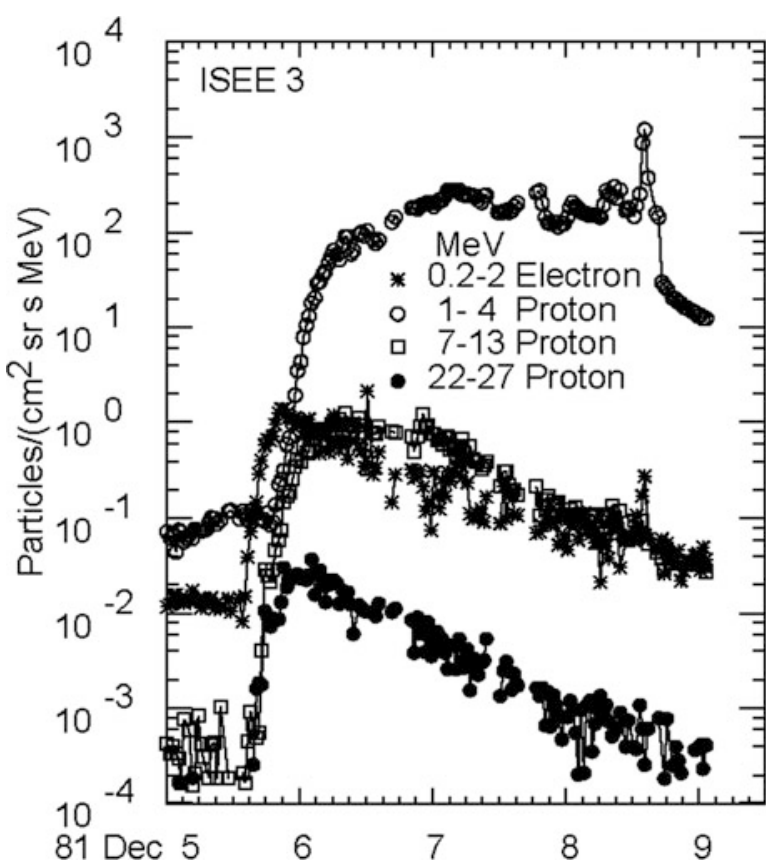

accelerate, so that a shock wave is not produced until $\sim 8 \mathrm{R}_{\mathrm{s}}$. Thus the properties of the SEPs are controlled by properties of the CME and the shock. However, flares are not required for SEP acceleration.

In contrast with these SEP events without flares, there are also "confined flares," X-class flares that have no CMEs or external SEPs (Gopalswamy et al. 2009).

\section{8 "The Solar-Flare Myth"}

By 1993, the idea of impulsive and gradual SEP events was fairly well documented, CMEs and CME-driven shocks had been studied for a decade in relation to SEPs, and ${ }^{3} \mathrm{He}$-rich events had been studied for two decades. It became increasingly clear that the largest SEP events (and the only ones producing a significant radiation hazards) were gradual events related to CMEs and shocks, not to flares. The birdcage model (Sect. 2.3.1) was dead. While reviews of this emerging paradigm were fairly common in invited talks at meetings, it was the publication of the review "The Solar Flare Myth" by Gosling (1993) that drew enormous criticism that surprised the SEP community. This fairly straightforward review was thought to "wage an assault on the last 30 years of solar-flare research" (Zirin 1994) by a flare community that usually ignored SEPs entirely. Apparently there was concern that if hazardous SEPs did not come from flares, flare research might be discontinued! The sky was falling! In hindsight, surely the last 25 years have proven such concerns to be unfounded. 
Unfortunately, however, there is still some visceral reluctance to embrace the idea of shock acceleration of SEPs, especially the shocks that produce GLEs.

The controversy raised by the Gosling (1993) paper led to an invited discussion from three alternative viewpoints in Eos where Hudson (1995) argued that the term "flare" should include the CME, shock, and any related physics, Miller (1995) argued that flares, being more numerous, were a better subject for acceleration studies, and Reames (1995) argued for the separate study of the physics of both flare and shock acceleration of SEPs. While the extension of the term "flare" has some philosophical merit, it is important for SEP studies to distinguish a pointsource flare or now, a localized jet, from the acceleration source at a broadlyextensive, Sun-spanning, CME-driven shock wave, especially when they involve different physical mechanisms.

\subsection{Wave Generation and the Streaming Limit}

When intensities of particles streaming along $\boldsymbol{B}$ are sufficiently great, they can amplify resonant Alfvén waves that exist or even generate them anew (Stix 1992; Melrose 1980). When in resonance, circularly polarized waves can maintain the orientation of their fields with respect to the velocity vector of the gyrating ions, maximizing the interaction. Systematic scattering of streaming ions reduces their energy only slightly but energy is conserved by amplifying the waves. These waves increase scattering and, in the vicinity of shock waves, increase acceleration. We have mentioned the early study of equilibrium wave growth and shock acceleration (Lee 1983). Here, waves are amplified upstream to compensate for those that are being swept into the shock. In fact, for simplicity, Lee assumed that $\mu=1$ so that $k \approx B / P$, i.e. each wave vector couples to its own single particle rigidity. When we allow $k \approx B / P \mu$, the waves can couple particles of different rigidity, an extremely important factor for many phenomena we observe.

Reames (1990) observed that 3-6 MeV proton intensities early in large gradual events never seemed to exceed a plateau value of $\sim 100-200\left(\mathrm{~cm}^{2} \mathrm{sr} \mathrm{s} \mathrm{MeV}\right)^{-1}$, subsequently called the "streaming limit," although intensities could rise much higher as the shock approached (see Fig. 5.3). Ng and Reames (1994) began by comparing transport with and without wave growth. They found that wave growth throttles the flow of particles, trapping them near the source, limiting their streaming. $\mathrm{Ng}$ et al. $(1999,2003,2012)$ extended these calculations showing how the scattering varied greatly in time and space, affecting $\mathrm{H}, \mathrm{He}, \mathrm{O}$, and $\mathrm{Fe}$ differently. The wave generation modifies the "initial" abundances seen early in SEP events (e.g. Reames et al. 2000). Further observations extended the streaming limit to higher energies (Reames and Ng 1998) and showed how the low-energy spectra can be flattened, but only when sufficient intensities of streaming high-energy protons precede them (Reames and $\mathrm{Ng}$ 2010). Wave growth and the streaming limit will be considered in detail in Sects. 5.1.2 and 5.1.5. 


\subsection{SEP-CME Correlation}

In his article on "the big-flare syndrome," Kahler (1982) pointed out that the fact that big SEP events are usually accompanied by big flares, does not mean that flares cause SEP events; rather, in larger events, all energetic phenomena may be more energetic or intense, including flares, CMEs, and SEPs. Flares were once incorrectly thought to cause CMEs. When there is a large rearrangement of the coronal magnetic field, much of the energy released is actually carried away by the CME (e.g. Emslie et al. 2004). Flares are not required to accompany CMEs or SEP events and are, in fact, a secondary phenomenon (Kahler 1992). When flares do accompany CMEs, the CME can precede the flare. Kahler (1992) asks "how did we form such a fundamentally incorrect view?" Probably, correlations of the other phenomena with familiar highly-visible flares were taken much too seriously.

While correlations do not necessarily imply a causal relationship, they are a starting point, and there is a steep dependence of peak particle intensity in large gradual SEP events on CME speed as shown in Fig. 2.12 (Kahler 2001). Two

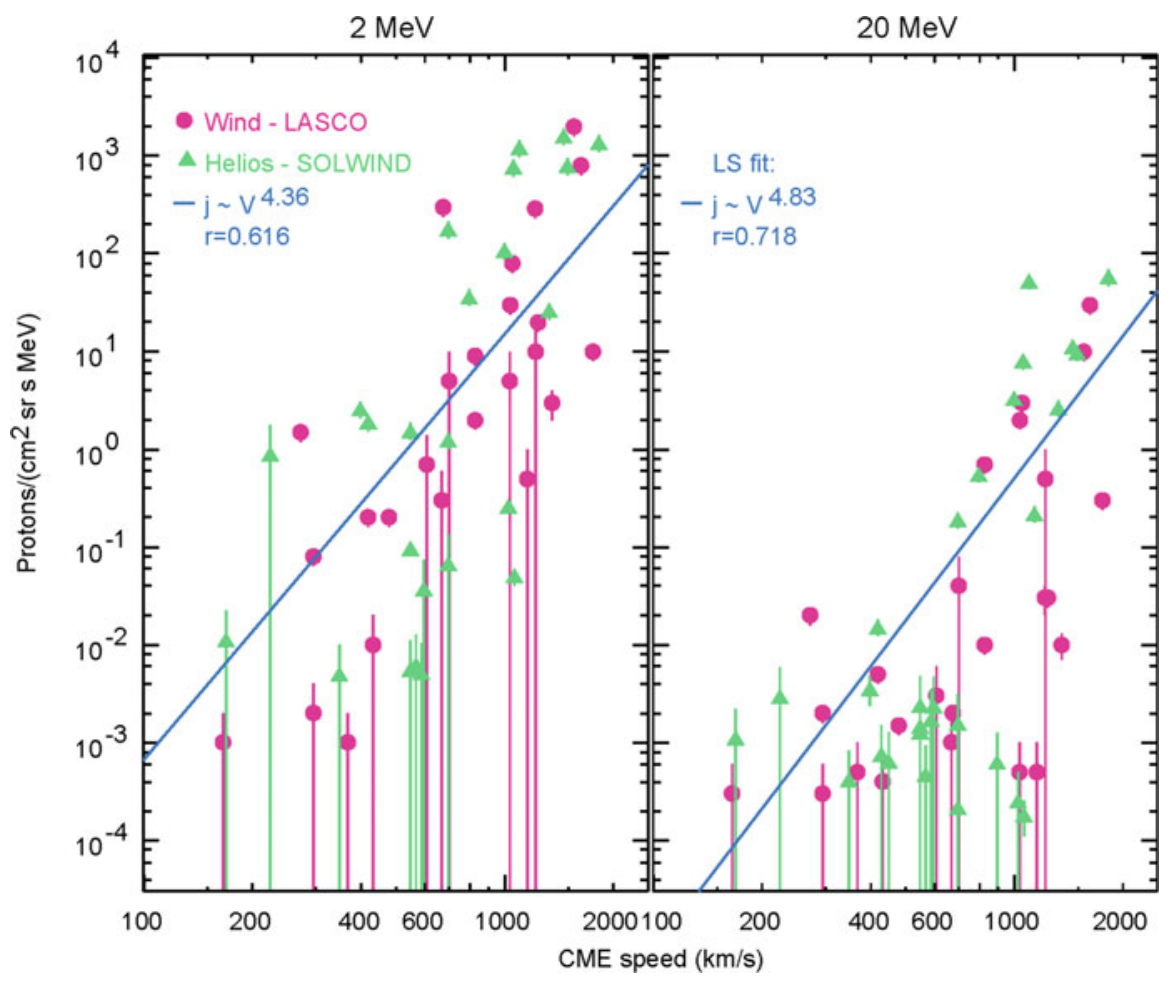

Fig. 2.12 Peak intensity is shown vs. CME speed for $2 \mathrm{MeV}$ (left) and $20 \mathrm{MeV}$ (right) protons for two event samples (see text). Power-law least-squares fits and correlation coefficients $(r)$ are shown (see Kahler 2001) 
samples of events are shown in the figure (1) SEPs measured on Wind and CMEs by SOHO/LASCO, both near Earth, and (2) SEPs measured on Helios, off the solar limbs, while the Naval Research Laboratory's Solwind coronagraph measured CMEs, from near Earth. The latter was an effort to correct for the projection effect in the direction of CME propagation. Of course the "peak intensity" is, in reality, a strong function of longitude, as expected from Fig. 2.2 (see also Fig. 5.16), as is the speed of the shock driven by the CME; these factors contribute to the spread of the measurement which, as we will see, may be reduced by using the measurements of multiple spacecraft in a single SEP event (see Fig. 3.4).

Recently, Kouloumvakos et al. (2019) have greatly improved the CME-SEP relationship by modeling the full $3 \mathrm{D}$ geometry of shock waves using the three coronagraph images from SOHO/LASCO and STEREO A and B. The peak proton intensities in three energy intervals from $20-100 \mathrm{MeV}$, on the three spacecraft, were then correlated with shock properties at the base of their magnetic flux tubes. The best correlation was 40-60 MeV protons with the Alfvén Mach number, which exceeded $75 \%$. Here a single 3D model of a shock predicted three SEP observations, each separated spatially by approximately $120^{\circ}$ for a total of 84 observations.

The apparent dependence on CME speed in Fig. 2.12 is certainly quite steep, although there is no physical reason that the relationship should be a power law. Fast CMEs are surely required to produce significant SEP events as originally suggested by Kahler et al. (1984). However, this type of correlation is only a basis for further study, and must be tested and improved as we will see in Sect. 3.2. What variables, other than CME speed, contribute to SEP intensities? Much of the remaining spread must be due to differences in particle transport conditions that spread the particles in space and produce time variations and delays in reaching peak intensities.

\subsection{SEPs Actually Cause Flares, Not the Reverse}

A recent study of the global energy distribution in flares and CMEs (Aschwanden et al. 2019) found that, of the magnetic energy released in reconnection in loops, $51 \pm 17 \%$ led to acceleration of electrons and $17 \pm 17 \%$ led to acceleration of ions. Despite the unusual error, measurements of $\gamma$-rays and neutrons (Sect. 4.9) suggest that $\mathrm{e} / \mathrm{p}$ ratios in flares are not unlike those in impulsive SEP events, and the essential point here is that $\sim$ half of the magnetic energy released is directly carried away by confined SEPs. These SEPs are scattered into the denser footpoints of the loops where they produce heating and evaporation of the plasma; this secondary thermal energy is dissipated as white light, UV, and soft X-ray emission, i.e. a hot, bright flare. Flares occur because the reconnection occurs on closed magnetic loops which the SEPs are unable to escape; when the reconnection involves open field lines, jets are produced and impulsive SEPs are seen in space. 
We tend to think of SEPs as particles measurable in space, but the energetic particles accelerated in closed magnetic-reconnection sites certainly deserve the name SEPs, and are the intermediary between the magnetic islands of reconnection and the sudden burst of heat and light we call a flare.

\section{References}

Aschwanden, M.J., Caspi, A., Cohen, C.M.S., Holman, G., Jing, J., Kretzschmar, M., Kontar, E.P., McTiernan, J.M., Mewaldt, R.A., O'Flannagain, A., Richardson, I.G., Ryan, D., Warren, H.P., $\mathrm{Xu}$, Y.: Global energetics of solar flares and coronal mass ejections. J. Phys. Conf. Ser. 1332, 1 (2019). https://doi.org/10.1088/1742-6596/1332/1/012002

Bertsch, D.L., Fichtel, C.E., Reames, D.V.: Relative abundance of iron-group nuclei in solar cosmic rays. Astrophys. J. Lett. 157, L53 (1969). https://doi.org/10.1086/180383

Bougeret, J.-L., Kaiser, M.L., Kellogg, P.J., et al.: WAVES: the radio and plasma wave investigation on the Wind spacecraft. Space Sci. Rev. 71, 5 (1995). https://doi.org/10.1007/BF00751331

Breneman, H.H., Stone, E.C.: Solar coronal and photospheric abundances from solar energetic particle measurements. Astrophys. J. Lett. 299, L57 (1985). https://doi.org/10.1086/184580

Bučík, R.: ${ }^{3} \mathrm{He}$-rich solar energetic particles: solar sources. Space Sci. Rev. 216, 24 (2020). https:// doi.org/10.1007/s11214-020-00650-5

Bučík, R., Innes, D.E., Mall, U., Korth, A., Mason, G.M., Gómez-Herrero, R.: Multi-spacecraft observations of recurrent ${ }^{3} \mathrm{He}$-rich solar energetic particles. Astrophys. J. 786, 71 (2014). https:// doi.org/10.1088/0004-637X/786/1/71

Bučík, R., Innes, D.E., Chen, N.H., Mason, G.M., Gómez-Herrero, R., Wiedenbeck, M.E.: Longlived energetic particle source regions on the Sun. J. Phys. Conf. Ser. 642, 012002 (2015). https://doi.org/10.1088/1742-6596/642/1/012002

Bučík, R., Innes, D.E., Mason, G.M., Wiedenbeck, M.E., Gómez-Herrero, R., Nitta, N.: ${ }^{3}$ He-rich solar energetic particles in helical jets on the sun. Astrophys. J. 852, 76 (2018). https://doi.org/ $10.3847 / 1538-4357 / \mathrm{aa} 9 \mathrm{~d} 8 \mathrm{f}$

Cane, H.V., Kahler, S.W., Sheeley Jr., N.R.: Interplanetary shocks preceded by solar filament eruptions. J. Geophys. Res. 91, 13321 (1986). https://doi.org/10.1029/JA091iA12p13321

Cane, H.V., Reames, D.V., von Rosenvinge, T.T.: The role of interplanetary shocks in the longitude distribution of solar energetic particles. J. Geophys. Res. 93, 9555 (1988). https://doi.org/10. 1029/JA093iA09p09555

Carrington, R.C.: Description of a singular appearance seen in the Sun on 1 September, 1859. Mon. Not. Roy. Astron. Soc. 20, 13 (1860). https://doi.org/10.1093/mnras/20.1.13

Chen, N.H., Bučík, R., Innes, D.E., Mason, G.M.: Case studies of multi-day ${ }^{3}$ He-rich solar energetic particle periods. Astron. Astrophys. 580, 16 (2015). https://doi.org/10.1051/0004$6361 / 201525618$

Cliver, E.W.: The unusual relativistic solar proton events of 1979 august 21 and 1981 may 10. Astrophys. J. 639, 1206 (2006). https://doi.org/10.1086/499765

Cliver, E.W., Kahler, S.W., Shea, M.A., Smart, D.F.: Injection onsets of $2 \mathrm{GeV}$ protons, $1 \mathrm{MeV}$ electrons, and $100 \mathrm{keV}$ electrons in solar cosmic ray flares. Astrophys. J. 260, 362 (1982). https://doi.org/10.1086/160261

Cliver, E.W., Kahler, S.W., Reames, D.V.: Coronal shocks and solar energetic proton events. Astrophys. J. 605, 902 (2004). https://doi.org/10.1086/382651

Cook, W.R., Stone, E.C., Vogt, R.E.: Elemental composition of solar energetic particles. Astrophys. J. 279, 827 (1984). https://doi.org/10.1086/161953 
Decker, R.B.: Formation of shock-spike events at quasi-perpendicular shocks. J. Geophys. Res. 88, 9959 (1983). https://doi.org/10.1029/JA088iA12p09959

Desai, M.I., Mason, G.M., Dwyer, J.R., Mazur, J.E., Smith, C.W., Skoug, R.M.: Acceleration of ${ }^{3}$ He Nuclei at Interplanetary Shocks. Astrophys. J. Lett. 553, L89 (2001). https://doi.org/10. $1086 / 320503$

Desai, M.I., Mason, G.M., Dwyer, J.R., Mazur, J.E., Gold, R.E., Krimigis, S.M., Smith, C.W., Skoug, R.M.: Evidence for a suprathermal seed population of heavy ions accelerated by interplanetary shocks near 1 AU. Astrophys. J. 588, 1149 (2003). https://doi.org/10.1086/ 374310

DiFabio, R., Guo, Z., Möbius, E., Klecker, B., Kucharek, H., Mason, G.M., Popecki, M.: Energydependent charge states and their connection with ion abundances in impulsive solar energetic particle events. Astrophys. J. 687, 623 (2008). https://doi.org/10.1086/591833

Drake, J.F., Cassak, P.A., Shay, M.A., Swisdak, M., Quataert, E.: A magnetic reconnection mechanism for ion acceleration and abundance enhancements in impulsive flares. Astrophys. J. Lett. 700, L16 (2009). https://doi.org/10.1088/0004-637X/700/1/L16

Ellison, D., Ramaty, R.: Shock acceleration of electrons and ions in solar flares. Astrophys. J. 298, 400 (1985). https://doi.org/10.1086/163623

Emslie, A.G., et al.: Energy partition in two solar flare/CME events. J. Geophys. Res. 109, A10104 (2004). https://doi.org/10.1029/2004JA010571

Fichtel, C.E., Guss, D.E.: Heavy nuclei in solar cosmic rays. Phys. Rev. Lett. 6, 495 (1961). https:// doi.org/10.1103/PhysRevLett.6.495

Fisk, L.A.: ${ }^{3}$ He-rich flares - a possible explanation. Astrophys. J. 224, 1048 (1978). https://doi.org/ $10.1086 / 156456$

Forbush, S.E.: Three unusual cosmic ray increases possibly due to charged particles from the Sun. Phys. Rev. 70, 771 (1946). https://doi.org/10.1103/PhysRev.70.771

Ganse, U., Kilian, P., Vainio, R., Spanier, F.: Emission of type II radio bursts - single-beam versus two-beam scenario. Sol. Phys. 280, 551 (2012). https://doi.org/10.1007/s11207-012-0077-7

Giacalone, J.: Particle acceleration at shocks moving through an irregular magnetic field. Astrophys. J. 624, 765 (2005). https://doi.org/10.1086/429265

Giaclaone, J., Jokipii, J.R.: The longitudinal transport of energetic ions from impulsive solar flares in interplanetary space. Astrophys J. Lett. 751, L33 (2012). https://doi.org/10.1088/2041-8205/ 751/2/L33

Gloeckler, G., Hovestadt, D., Vollmer, O., Fan, C.Y.: Unusual emission of iron nuclei from the sun. Astrophys J. Lett. 200, L45 (1975). https://doi.org/10.1086/181893

Gopalswamy, N., Yashiro, S., Michalek, G., Kaiser, M.L., Howard, R.A., Reames, D.V., Leske, R., von Rosenvinge, T.: Interacting coronal mass ejections and solar energetic particles. Astrophys. J. (Letters). 572, L103 (2002). https://doi.org/10.1086/341601

Gopalswamy, N., Akiyama, S., Yashiro, S.: Major solar flares without coronal mass ejections. In: Gopalswamy, N., Webb, D.F. (eds.) Proceedings of the IAU symposium, vol. 257, p. 283 (2009). https://doi.org/10.1017/S174392130902941X

Gopalswamy, N., Xie, H., Yashiro, S., Akiyama, S., Mäkelä, P., Usoskin, I.G.: Properties of Ground level enhancement events and the associated solar eruptions during solar cycle 23. Space Sci. Rev. 171, 23 (2012). https://doi.org/10.1007/s11214-012-9890-4

Gopalswamy, N., Mäkelä, P., Akiyama, S., Yashiro, S., Xie, H., Thakur, N., Kahler, S.W.: Large solar energetic particle events associated with filament eruptions outside of active regions. Astrophys. J. 806, 8 (2015). https://doi.org/10.1088/0004-637X/806/1/8

Gosling, J.T.: The solar flare myth. J. Geophys. Res. 98, 18937 (1993). https://doi.org/10.1029/ 93JA01896

Ho, G.C., Roelof, E.C., Mason, G.M.: The upper limit on ${ }^{3} \mathrm{He}$ fluence in solar energetic particle events. Atrophys. J. Lett. 621, L141 (2005). https://doi.org/10.1086/429251 
Hsieh, K.C., Simpson, J.A.: The relative abundances and energy spectra of ${ }^{3} \mathrm{He}$ and ${ }^{4} \mathrm{He}$ from solar flares. Astrophys. J. Lett. 162, L191 (1970). https://doi.org/10.1086/180652

Hudson, H.S.: Solar flares: No "myth". Eos. Trans. AGU. 76(41), 405 (1995). https://doi.org/10. 1029/95EO00253

Ibragimov, I.A., Kocharov, G.E.: Possible mechanism for enrichment of solar cosmic rays by helium-three and heavy nuclei. 15th International conference on cosmic rays (Plovdiv: Bulgarian Academy of Sciences), 11, 340 (1977)

Jokipii, J.R., Parker, E.N.: Stochastic aspects of magnetic lines of force with application to cosmic ray propagation. Astrophys. J. 155, 777 (1969). https://doi.org/10.1086/149909

Jones, F.C., Ellison, D.E.: The plasma physics of shock acceleration. Space Sci. Rev. 58, 259 (1991). https://doi.org/10.1007/BF01206003

Kahler, S.W.: The role of the big flare syndrome in correlations of solar energetic proton fluxes and associated microwave burst parameters. J. Geophys. Res. 87, 3439 (1982). https://doi.org/10. 1029/JA087iA05p03439

Kahler, S.W.: Solar flares and coronal mass ejections. Annu. Rev. Astron. Astrophys. 30, 113 (1992). https://doi.org/10.1146/annurev.aa.30.090192.000553

Kahler, S.W.: The correlation between solar energetic particle peak intensities and speeds of coronal mass ejections: Effects of ambient particle intensities and energy spectra. J. Geophys. Res. 106, 20947 (2001). https://doi.org/10.1029/2000JA002231

Kahler, S.W., Sheeley Jr., N.R., Howard, R.A., Koomen, M.J., Michels, D.J., McGuire, R.E., von Rosenvinge, T.T., Reames, D.V.: Associations between coronal mass ejections and solar energetic proton events. J. Geophys. Res. 89, 9683 (1984). https://doi.org/10.1029/ JA089iA11p09683

Kahler, S.W., Cliver, E.W., Cane, H.V., McGuire, R.E., Stone, R.G., Sheeley Jr., N.R.: Solar filament eruptions and energetic particle events. Astrophys. J. 302, 594 (1986). https://doi.org/ $10.1086 / 164009$

Kahler, S.W., Reames, D.V., Sheeley Jr., N.R.: Coronal mass ejections associated with impulsive solar energetic particle events. Astrophys. J. 562, 558 (2001). https://doi.org/10.1086/323847

Kocharov, G. E., Kocharov, L. G.: Present state of experimental and theoretical investigations of solar events enriched by helium-3. In: Proceedings of the 10th Leningrad Symposium on Cosmic Rays (Leningrad: A. F. Yoffe Phys.-Tech. Inst.), p. 37 (1978)

Kocharov, G.E., Kocharov, L.G.: ${ }^{3}$ He-rich solar flares. Space Sci. Rev. 38, 89 (1984). https://doi. org/10.1007/BF00180337

Kouloumvakos, A., Rouillard, A., Wu, Y., Vainio, R., Vourlidas, A., Plotnikov, I., Afanasiev, A., Önel, H.: Connecting the properties of coronal shock waves with those of solar energetic particles. Astrophys. J. 876, 80 (2019). https://doi.org/10.3847/1538-4357/ab15d7

Laming, J.M.: A unified picture of the first ionization potential and inverse first ionization potential effects. Astrophys. J. 614, 1063 (2004). https://doi.org/10.1086/423780

Laming, J.M.: Non-WKB models of the first ionization potential effect: implications for solar coronal heating and the coronal helium and neon abundances. Astrophys. J. 695, 954 (2009). https://doi.org/10.1088/0004-637X/695/2/954

Lee, M.A.: Coupled hydromagnetic wave excitation and ion acceleration at interplanetary traveling shocks. J. Geophys. Res. 88, 6109 (1983). https://doi.org/10.1029/JA088iA08p06109

Lee, M.A.: Coupled hydromagnetic wave excitation and ion acceleration at an evolving coronal/ interplanetary shock. Astrophys. J. Suppl. 158, 38 (2005). https://doi.org/10.1086/428753

Leske, R.A., Cummings, J.R., Mewaldt, R.A., Stone, E.C., von Rosenvinge, T.T.: Measurements of the ionic charge states of solar energetic particles using the geomagnetic field. Astrophys. J. 452, L149 (1995). https://doi.org/10.1086/309718

Li, G., Zank, G.P., Verkhoglyadova, O., Mewaldt, R.A., Cohen, C.M.S., Mason, G.M., Desai, M.I.: Shock geometry and spectral breaks in large sep events. Astrophys. J. 702, 998 (2009). https:// doi.org/10.1088/0004-637X/702/2/998 
Lin, R.P.: The emission and propagation of $40 \mathrm{keV}$ solar flare electrons. I: the relationship of $40 \mathrm{keV}$ electron to energetic proton and relativistic electron emission by the sun. Sol. Phys. 12, 266 (1970). https://doi.org/10.1007/BF00227122

Lin, R.P.: Non-relativistic solar electrons. Space Sci. Rev. 16, 189 (1974). https://doi.org/10.1007/ BF00240886

Litvinenko, Y.E.: On the formation of the helium-3 spectrum in impulsive solar flares. In: Ramaty, R., Mandzhavidze, N., Hua, X.-M. (eds.) High energy solar physics, AIP conference proceedings, vol. 374, p. 498. AIP Press, Woodbury, NY (1996). https://doi.org/10.1063/1. 50985

Luhn, A., Klecker, B., Hovestadt, D., Gloeckler, G., Ipavich, F.M., Scholer, M., Fan, C.Y., Fisk, L. A.: Ionic charge states of $\mathrm{N}, \mathrm{Ne}, \mathrm{Mg}, \mathrm{Si}$ and $\mathrm{S}$ in solar energetic particle events. Adv. Space Res. 4, 161 (1984). https://doi.org/10.1016/0273-1177(84)90307-7

Luhn, A., Klecker, B., Hovestadt, D., Möbius, E.: The mean ionic charge of silicon in He-3-rich solar flares. Astrophys. J. 317, 951 (1987). https://doi.org/10.1086/165343

Mason, G.M., Gloeckler, G., Hovestadt, D.: Temporal variations of nucleonic abundances in solar flare energetic particle events. II - Evidence for large-scale shock acceleration. Astrophys. J. 280, 902 (1984). https://doi.org/10.1086/162066

Mason, G.M., Reames, D.V., Klecker, B., Hovestadt, D., von Rosenvinge, T.T.: The heavy-ion compositional signature in He-3-rich solar particle events. Astrophys. J. 303, 849 (1986). https:// doi.org/10.1086/164133

Mason, G.M., Ng, C.K., Klecker, B., Green, G.: Impulsive acceleration and scatter-free transport of about $1 \mathrm{MeV}$ per nucleon ions in ${ }^{3} \mathrm{He}$-rich solar particle events. Astrophys. J. 339, 529 (1989). https://doi.org/10.1086/167315

Mason, G.M., Mazur, J.E., Dwyer, J.R.: ${ }^{3} \mathrm{He}$ enhancements in large solar energetic particle events. Astrophys. J. Lett. 525, L133 (1999). https://doi.org/10.1086/312349

Melrose, D.B.: Plasma astrophysics, vol. 1. Gordon and Breach, New York (1980)

Meyer, J.P.: The baseline composition of solar energetic particles. Astrophys. J. Suppl. 57, 151 (1985). https://doi.org/10.1086/191000

Meyer, P., Parker, E.N., Simpson, J.A.: Solar cosmic rays of February, 1956 and their propagation through interplanetary space. Phys. Rev. 104, 768 (1956). https://doi.org/10.1103/PhysRev.104. 768

Miller, J.A.: Much ado about nothing. Eos. Trans. AGU. 76(41), 401 (1995). https://doi.org/10. 1029/95EO00246

Miller, J., Viñas, A., Reames, D.V.: Selective ${ }^{3} \mathrm{He}$ and Fe acceleration in impulsive solar flares. 23rd International Cosmic-Ray Conference (Calgary). 3, 13 (1993a)

Miller, J., Viñas, A., Reames, D.V.: Heavy ion acceleration and abundance enhancements in impulsive solar flares. 23rd International Cosmic-Ray Conference (Calgary). 3, 17 (1993b)

Newkirk Jr., G., Wenzel, D.G.: Rigidity-independent propagation of cosmic rays in the solar corona. J. Geophys. Res. 83, 2009 (1978). https://doi.org/10.1029/JA083iA05p02009

$\mathrm{Ng}$, C.K., Reames, D.V.: Focused interplanetary transport of approximately $1 \mathrm{MeV}$ solar energetic protons through self-generated Alfven waves. Astrophys. J. 424, 1032 (1994). https://doi.org/ $10.1086 / 173954$

$\mathrm{Ng}$, C.K., Reames, D.V.: Shock acceleration of solar energetic protons: the first 10 minutes. Astrophys. J. Lett. 686, L123 (2008). https://doi.org/10.1086/592996

Ng, C.K., Reames, D.V., Tylka, A.J.: Effect of proton-amplified waves on the evolution of solar energetic particle composition in gradual events. Geophys. Res. Lett. 26, 2145 (1999). https:// doi.org/10.1029/1999GL900459

Ng, C.K., Reames, D.V., Tylka, A.J.: Modeling shock-accelerated solar energetic particles coupled to interplanetary Alfvén waves. Astrophys. J. 591, 461 (2003). https://doi.org/10.1086/375293

Ng, C.K., Reames, D.V., Tylka, A.J.: Solar energetic particles: shock acceleration and transport through self-amplified waves. AIP Conference Proceedings. 1436, 212 (2012). https://doi.org/ $10.1063 / 1.4723610$ 
Nitta, N.V., Reames, D.V., DeRosa, M.L., Yashiro, S., Gopalswamy, N.: Solar sources of impulsive solar energetic particle events and their magnetic field connection to the earth. Astrophys. J. 650, 438 (2006). https://doi.org/10.1086/507442

Reames, D.V.: Acceleration of energetic particles by shock waves from large solar flares. Astrophys. J. Lett. 358, L63 (1990). https://doi.org/10.1086/185780

Reames, D.V.: The dark side of the solar flare myth. Eos. Trans. AGU. 76(41), 401 (1995). https:// doi.org/10.1029/95EO00254

Reames, D.V.: Particle acceleration at the sun and in the heliosphere. Space Sci. Rev. 90, 413 (1999). https://doi.org/10.1023/A:1005105831781

Reames, D.V.: Abundances of trans-iron elements in solar energetic particle events. Astrophys. J. Lett. 540, L111 (2000). https://doi.org/10.1086/312886

Reames, D.V.: Temperature of the source plasma in gradual solar energetic particle events. Sol. Phys. 291, 911 (2016). https://doi.org/10.1007/s11207-016-0854-9. arXiv: 1509.08948

Reames, D.V., Ng, C.K.: Streaming-limited intensities of solar energetic particles. Astrophys. J. 504, 1002 (1998). https://doi.org/10.1086/306124

Reames, D.V., Ng, C.K.: Streaming-limited intensities of solar energetic particles on the intensity plateau. Astrophys. J. 722, 1286 (2010). https://doi.org/10.1088/0004-637X/723/2/1286

Reames, D.V., Stone, R.G.: The identification of solar He-3-rich events and the study of particle acceleration at the sun. Astrophys. J. 308, 902 (1986). https://doi.org/10.1086/164560

Reames, D.V., von Rosenvinge, T.T., Lin, R.P.: Solar He-3-rich events and nonrelativistic electron events - a new association. Astrophys. J. 292, 716 (1985). https://doi.org/10.1086/163203

Reames, D.V., Meyer, J.P., von Rosenvinge, T.T.: Energetic-particle abundances in impulsive solar flare events. Astrophys. J. Suppl. 90, 649 (1994). https://doi.org/10.1086/191887

Reames, D.V., Barbier, L.M., Ng, C.K.: The spatial distribution of particles accelerated by coronal mass ejection-driven shocks. Astrophys. J. 466, 473 (1996). https://doi.org/10.1086/177525

Reames, D.V., Ng, C.K., Tylka, A.J.: Initial time dependence of abundances in solar particle events. Astrophys. J. Lett. 531, L83 (2000). https://doi.org/10.1086/312517

Reames, D.V., Cliver, E.W., Kahler, S.W.: Abundance enhancements in impulsive solar energeticparticle events with associated coronal mass ejections. Sol. Phys. 289, 3817 (2014a). https://doi. org/10.1007/s11207-014-0547-1

Reames, D.V., Cliver, E.W., Kahler, S.W.: Variations in abundance enhancements in impulsive solar energetic-particle events and related CMEs and flares. Sol. Phys. 289, 4675 (2014b). https://doi.org/10.1007/s11207-014-0589-4

Reid, G.C.: A diffusive model for the initial phase of a solar proton event. J. Geophys. Res. 69, 2659 (1964). https://doi.org/10.1029/JZ069i013p02659

Reinhard, R., Wibberenz, G.: Propagation of flare protons in the solar atmosphere. Sol. Phys. 36, 473 (1974). https://doi.org/10.1007/BF00151216

Richardson, I.G., Reames, D.V., Wenzel, K.-P., Rodriguez-Pacheco, J.: Quiet-time properties of low-energy (less than $10 \mathrm{MeV}$ per nucleon) interplanetary ions during solar maximum and solar minimum. Astrophys. J. Lett. 363, L9 (1990). https://doi.org/10.1086/185853

Riyopoulos, S.: Subthreshold stochastic diffusion with application to selective acceleration of ${ }^{3} \mathrm{He}$ in solar flares. Astrophys. J. 381, 578 (1991). https://doi.org/10.1086/170682

Roth, I., Temerin, M.: Enrichment of ${ }^{3} \mathrm{He}$ and heavy ions in impulsive solar flares. Astrophys. J. 477, 940 (1997). https://doi.org/10.1086/303731

Sanahuja, B., Domingo, V., Wenzel, K.-P., Joselyn, J.A., Keppler, E.: A large proton event associated with solar filament activity. Sol. Phys. 84, 321 (1983). https://doi.org/10.1007/ BF00157465

Sandroos, A., Vainio, R.: Simulation results for heavy ion spectral variability in large gradual solar energetic particle events. Astrophys. J. 662, L127 (2007). https://doi.org/10.1086/519378

Serlemitsos, A.T., Balasubrahmanyan, V.K.: Solar particle events with anomalously large relative abundance of ${ }^{3} \mathrm{He}$. Astrophys. J. 198, 195 (1975). https://doi.org/10.1086/153592 
Steinacker, J., Meyer, J.-P., Steinacker, A., Reames, D.V.: The helium valley: comparison of impulsive solar flare ion abundances and gyroresonant acceleration with oblique turbulence in a hot multi-ion plasma. Astrophys. J. 476, 403 (1997). https://doi.org/10.1086/303589

Stix, T.H.: Waves in plasmas. AIP, New York (1992)

Temerin, M., Roth, I.: The production of ${ }^{3} \mathrm{He}$ and heavy ion enrichment in ${ }^{3} \mathrm{He}$-rich flares by electromagnetic hydrogen cyclotron waves. Astrophys. J. Lett. 391, L105 (1992). https://doi. org/10.1086/186408

Thejappa, G., MacDowall, R.J., Bergamo, M., Papadopoulos, K.: Evidence for the oscillating two stream instability and spatial collapse of Langmuir waves in a solar type III radio burst. Astrophys. J. Lett. 747, L1 (2012). https://doi.org/10.1088/2041-8205/747/1/L1

Tylka, A.J., Dietrich, W.F.: A new and comprehensive analysis of proton spectra in ground-level enhanced (GLE) solar particle events. In: Proceedings of the 31st International Cosmic Ray Conference, Lódz (2009). http://icrc2009.uni.lodz.pl/proc/pdf/icrc0273.pdf

Tylka, A.J., Lee, M.A.: Spectral and compositional characteristics of gradual and impulsive solar energetic particle events. Astrophys. J. 646, 1319 (2006). https://doi.org/10.1086/505106

Tylka, A.J., Boberg, P.R., Adams Jr., J.H., Beahm, L.P., Dietrich, W.F., Kleis, T.: The mean ionic charge state of solar energetic Fe ions above $200 \mathrm{MeV}$ per nucleon. Astrophys. J. 444, L109 (1995). https://doi.org/10.1086/187872

Tylka, A.J., Cohen, C.M.S., Dietrich, W.F., Maclennan, C.G., McGuire, R.E., Ng, C.K., Reames, D.V.: Evidence for remnant flare suprathermals in the source population of solar energetic particles in the 2000 bastille day event. Astrophys. J. Lett. 558, L59 (2001). https://doi.org/10. $1086 / 323344$

Tylka, A.J., Cohen, C.M.S., Dietrich, W.F., Lee, M.A., Maclennan, C.G., Mewaldt, R.A., Ng, C.K., Reames, D.V.: Shock geometry, seed populations, and the origin of variable elemental composition at high energies in large gradual solar particle events. Astrophys. J. 625, 474 (2005). https://doi.org/10.1086/429384

Varvoglis, H., Papadopoulis, K.: Selective nonresonant acceleration of He-3(2+) and heavy ions by H(+) cyclotron waves. Astrophys. J. Lett. 270, L95 (1983). https://doi.org/10.1086/184077

Verkhoglyadova, O., Zank, G.P., Li, G.: A theoretical perspective on particle acceleration by interplanetary shocks and the Solar Energetic Particle problem. Phys. Rep. 557, 1 (2015). https://doi.org/10.1016/j.physrep.2014.10.004

Wang, Y.-M., Pick, M., Mason, G.M.: Coronal holes, jets, and the origin of ${ }^{3} \mathrm{He}$-rich particle events. Astrophys. J. 639, 495 (2006). https://doi.org/10.1086/499355

Weatherall, J.: Turbulent heating in solar cosmic-ray theory. Astrophys. J. 281, 468 (1984). https:// doi.org/10.1086/162119

Webber, W.R.: Solar and galactic cosmic ray abundances - a comparison and some comments. Proceedings of the 14th International Cosmic Ray Conference, Munich. 5, 1597 (1975)

Webber, W.R., Roelof, E.C., McDonald, F.B., Teegarden, B.J., Trainor, J.: Pioneer 10 measurements of the charge and energy spectrum of solar cosmic rays during 1972 August. Astrophys. J. 199, 482 (1975). https://doi.org/10.1086/153714

Wiedenbeck, M.E., Mason, G.M., Cohen, C.M.S., Nitta, N.V., Gómez-Herrero, R., Haggerty, D.K.: Observations of solar energetic particles from ${ }^{3} \mathrm{He}$-rich events over a wide range of heliographic longitude. Astrophys. J. 762, 54 (2013). https://doi.org/10.1088/0004-637X/762/1/54

Wild, J.P., Smerd, S.F., Weiss, A.A.: Solar Bursts. Annu. Rev. Astron. Astrophys. 1, 291 (1963). https://doi.org/10.1146/annurev.aa.01.090163.001451

Winglee, R.M.: Heating and acceleration of heavy ions during solar flares. Astrophys. J. 343, 511 (1989). https://doi.org/10.1086/167726

Zank, G.P., Rice, W.K.M., Wu, C.C.: Particle acceleration and coronal mass ejection driven shocks: a theoretical model. J. Geophys. Res. 105, 25079 (2000). https://doi.org/10.1029/ 1999JA000455 
Zank, G.P., Li, G., Verkhoglyadova, O.: Particle acceleration at interplanetary shocks. Space Sci. Rev. 130, 255 (2007). https://doi.org/10.1007/s11214-007-9214-2

Zhao, L., Zhang, M., Rassoul, H.K.: Double power laws in the event-integrated solar energetic particle spectrum. Astrophys. J. 821, 62 (2016). https://doi.org/10.3847/0004-637X/821/1/62

Zirin, H.: Solar storminess, Sky and Telescope, Nov, 9 (1994)

Open Access This chapter is licensed under the terms of the Creative Commons Attribution 4.0 International License (http://creativecommons.org/licenses/by/4.0/), which permits use, sharing, adaptation, distribution and reproduction in any medium or format, as long as you give appropriate credit to the original author(s) and the source, provide a link to the Creative Commons license and indicate if changes were made.

The images or other third party material in this chapter are included in the chapter's Creative Commons license, unless indicated otherwise in a credit line to the material. If material is not included in the chapter's Creative Commons license and your intended use is not permitted by statutory regulation or exceeds the permitted use, you will need to obtain permission directly from the copyright holder.

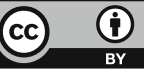

